\title{
Some Analytical Results About Two Physical Models of Phyllotaxis
}

\author{
M. Kunz \\ Institut de Physique Expérimentale, Université de Lausanne, CH-1015 Lausanne, Switzerland \\ Received: 17 January 1994/in revised form: 7 July 1994
}

\begin{abstract}
We present analytical results about a generalization of the variational problem of Levitov and the experiment of Douady and Couder. We show that the hierarchical selection of noble numbers in these systems occurs with a precision depending on the range of the interaction. This precision can be infinite if the interaction is scale invariant.
\end{abstract}

\section{Introduction}

1.1 Phyllotaxis, or the Enigma of Botanical Patterns. Let us first of all give a brief survey of a problem of growth and form in botany known as phyllotaxis. It is in fact the starting point of the problematics discussed below.

If one looks at the seeds of a sunflower, the florets in a daisy or the scales of a pineapple, one is struck not only by the regular lattice they form, but also by the nearly systematic appearance of Fibonacci numbers in these patterns (try counting the number of spirals connecting nearest neighbours in the spiral lattice shown in Fig. 1).

Early botanical observations showed that the regularity of the phyllotactic pattern is directly related to the cellular differentiation process [1,2]. Each budding leaf (or scale, or floret) appears on a ring shaped region (the meristem) surrounding the top of the stem. As the plant grows, the leaves are advected away radially from the meristem. The macroscopic structure of the leaves is therefore determined by this initial microscopic process.

Elementary geometry and number theory show that a regular lattice of leaves is created if the angular distance $2 \pi \theta$ between two consecutive leaves on the meristem is approximately constant. $\theta \in[0,1]$ is called the divergence of the lattice. Moreover Fibonacci numbers arise when the divergence is a good approximation of the Fibonacci or golden divergence [3-5]:

$$
\theta_{F}=\tau^{-2}=0.381966 \ldots
$$




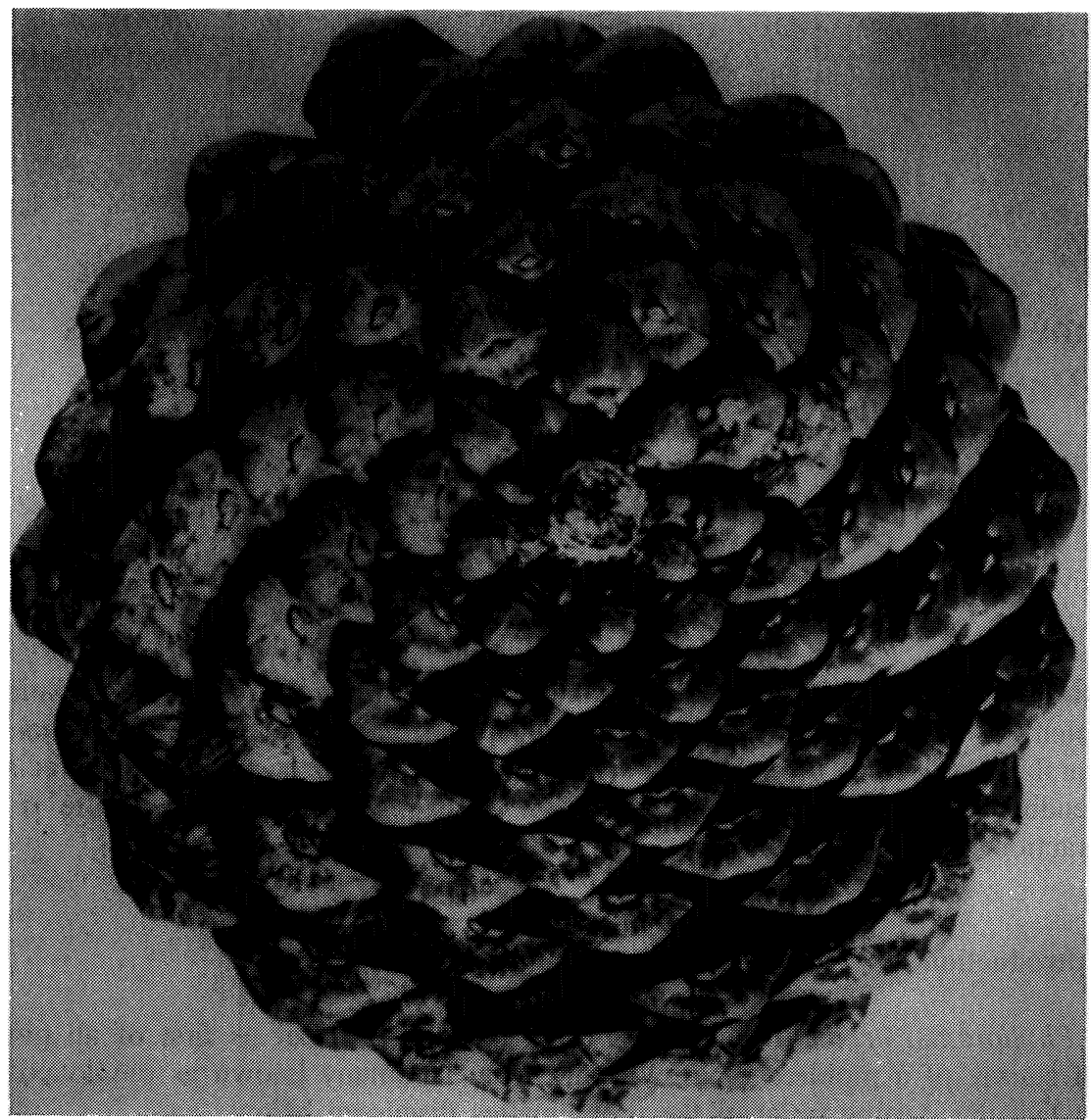

Fig. 1. One can distinguish two families of spirals (left-hand and right-hand) connecting adjacent scales of this fir cone. The numbers of spirals in each family turn out to be two successive integers in the Fibonacci sequence:

$$
1,1,2,3,5,8,13,21, \ldots
$$

Here $\tau$ is the famous golden mean, defined as

$$
\tau=\frac{\sqrt{5}+1}{2}
$$

The fascination exerted by this irrational number and its remarkable arithmetical properties dates back to antiquity. In particular, the continued fraction expansion of $\theta_{F}$ is extremely simple:

$$
\theta_{F}=\frac{1}{2+\frac{1}{1+\frac{1}{1+\ldots .}}}
$$

Generally speaking the divergences whose continued fraction development finishes with an infinite sequence of units are called noble numbers. They are of special interest in phyllotaxis. 
A first question to ask is whether the appearance of Fibonacci numbers or, more generally, of noble numbers as divergences, is due to some geometrical constraints. For if we represent the set of leaves by a lattice of points, it is possible to centre nonoverlapping tangent circles at each point only if the lattice fulfills some conditions. Let us represent for instance cylindrical phyllotactic patterns by a two-parameter family of plane periodic lattices. The set of lattices for which the construction of a lattice of tangent circles of constant radius is possible corresponds to a tree in the parameter plane (see Fig. 2). We will describe later the remarkable properties of such a tree, first obtained by Van Iterson [7]. Let us mention however that there is exactly one path in the tree leading to any irrational divergence on the axis $G=0$. In this sense all divergences are equally possible and the question of the appearance of Fibonacci numbers in nature still remains unresolved.

What happens if the hard-core interaction between leaves introduced by tangent circles is replaced by an arbitrary repulsive potential? How can we modify in this case the geometrical constraint of tangency of circles?

Recently Levitov [8,9] and Douady and Couder [10] gave an answer to these questions. They studied two physical systems, either theoretically or experimentally, whose main characteristic, as they emphasize, is a hierarchical selection of noble numbers. As a matter of fact, each bifurcation in the tree of Fig. 2 is replaced by a quasi-bifurcation, so that only a countable set of paths is left. Levitov as well as Douady and Couder pointed out the analogy of these systems with phyllotaxis. The aim of this paper is to give some theorems concerning the selection of noble numbers in these problems.

\subsection{The Flux Lattice of Levitov and the Experiment of Douady and Couder.} Inspired by geometrical models of phyllotaxis, Levitov considered a flux lattice pinned by superconducting layers [8]. Both the magnetic field and the vortices are

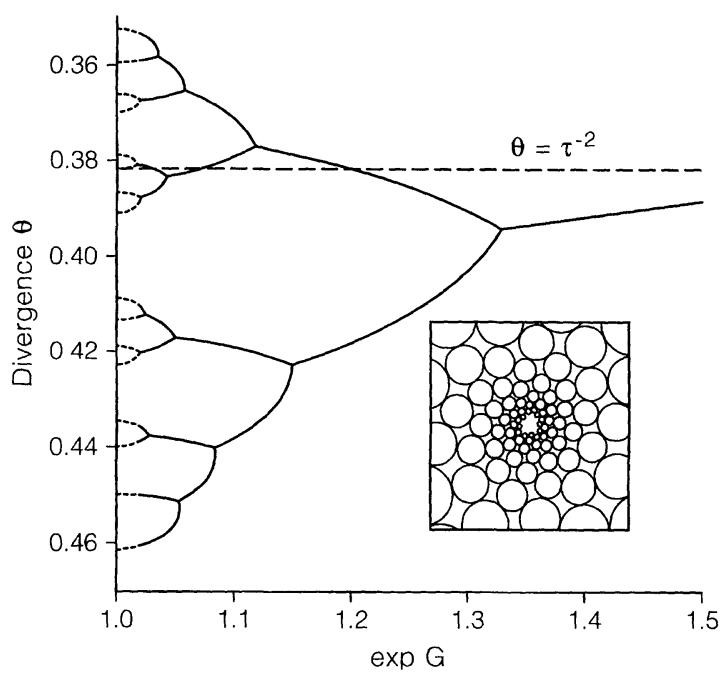

Fig. 2. Packing of tangent circles on a periodic lattice (or along a logarithmic spiral) corresponds to a purely geometrical constraint. The subset constituted by the lattices for which this construction is possible, is a tree in the parameter plane (reproduced with permission from [6]) 
parallel to the layers. Since the vortices interact repulsively at all distances, the lattice is assumed to be simply periodic. In the model proposed by Levitov, the energy of a flux lattice is

$$
E(G, \theta)=\sum_{m \in \mathbb{Z}} \sum_{n \geqq 1} u\left(\left\|\mathbf{r}_{n m}\right\|\right)
$$

where

$$
\mathbf{r}_{n m}=(n \theta-m) \mathbf{e}_{x}+n G \mathbf{e}_{y}
$$

and $u$ is some repulsive potential. $G$ is a control parameter which can be made to vary by changing the applied magnetic field. $\theta$ is the analogous of the divergence in a phyllotactic pattern.

Levitov tackled the mathematical problem of describing the set of couples $(G, \theta)$ such that $\theta$ is a relative minimum of $E(G, \theta)$ for a fixed value of $G$ (we will refer to this set later as the divergence spectrum). The numerical plots he presented show a remarkable tree structure, each branch approaching a noble number $\theta$ on the axis $G=0$ according to the hierarchical rule of Farey ${ }^{1}$ (see Fig. 3). In his analysis, Levitov brought to light the hidden symmetry of this particular problem [9]: if one puts $\mathbf{r}_{n m} / \sqrt{G}$ in place of $\mathbf{r}_{n m}$ in $(1.1)$, then $E(G, \theta)=E\left(G^{\prime}, \theta^{\prime}\right)$, where $\left(G^{\prime}, \theta^{\prime}\right)$ is the image of $(G, \theta)$ by some homographic transformation. These transformations form the group $S L(2, \mathbb{Z}) \otimes \mathbb{Z}_{2}$.

Levitov does not set a theorem specifying the class of potentials $u(r)$ for which the divergence spectrum has this remarkable structure. Nevertheless he develops a very nice argument which rests on the symmetry of the problem and must therefore be valid for almost any smooth potential.

Many authors also developed a dynamic approach to phyllotaxis $[1,10-17]$. In order to account for the appearance of the new leaves on the meristem, they postulated a simple discrete-time mechanism: suppose that each existing leaf exerts an inhibiting action on the meristem. The older the leaf is, the weaker its action is. The new leaf is positioned on the meristem at the minimum of the resulting inhibiting action. This mechanism produces stationary solutions; in other words, the angular distance between two consecutive leaves can stabilize after some time.

A physical realization of this mechanism has been made by Douady and Couder [10]. In their experiment, particles interacting through a repulsive potential $u(r)$ periodically fall on a cone situated at the center of a dish. The particles are then advected away radially in the dish, which is filled with a viscous fluid. Insofar as the interaction between the advected particles is negligible, this is exactly the dynamic scheme defined above: a new particle (representing a leaf) reaches the boundary of the central cone (the meristem) at the minimum of the repulsive potential created by previous particles.

In order to simulate numerically this experiment, Douady and Couder defined an iterative dynamical system which incorporates the typical features of the physical system. Lay $N$ particles on the plane outside of a unit disk centered at the origin, and associate to each of them a repulsive potential $u(r)$. Let $\mathbf{r}_{1}, \ldots, \mathbf{r}_{N}$ be the position of the particles, and let $U(\phi)$ be the resulting potential energy on the unit circle, $\phi$ being an angular coordinate. A new particle is placed on the unit circle at the

1 The physical meaning of this problem is not very clear. Levitov claims that if one varies $G$ adiabatically from $+\infty$ to 0 , the divergence $\theta$ will follow the continuous line of the spectrum that starts at $(G=+\infty, \theta=0.5)$ and ends at $(G=0, \theta=0.382 .$.$) . However, the condition " \theta$ is a relative minimum of $E(G=$ const, $\theta)$ " is not a criterion of stability of the lattice $(G, \theta)$. 

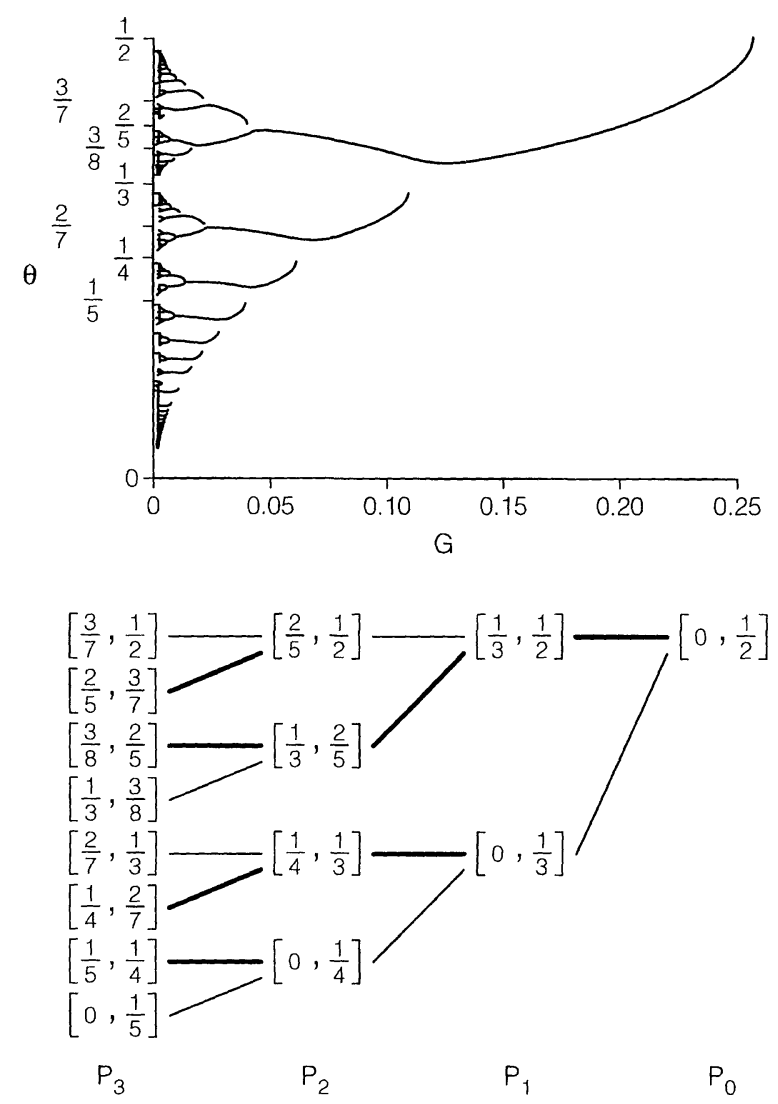

Fig. 3. Showing a typical solution of the problem of Levitov. Compare its structure with the Farey tree below. The Farey tree is a systematic way of constructing real numbers, that is the closure of the set of rational numbers. A partition $P_{n}$ of $[0,1 / 2]$ in $2^{n}$ intervals is obtained from $P_{n-1}$ by associating a regular interval $\left[\frac{p}{q}, \frac{p+p^{\prime}}{q+q^{\prime}}\right]$ and a singular interval $\left[\frac{p+p^{\prime}}{q+q^{\prime}}, \frac{p^{\prime}}{q^{\prime}}\right]$ to each interval $\left[\frac{p}{q}, \frac{p^{\prime}}{q^{\prime}}\right] \in P_{n-1}\left(q>q^{\prime}\right)$. The thick lines lead to regular intervals.

In the spectrum of Levitov, each branch oscillates so that $\theta$ belongs to successive regular intervals of the Farey tree when $G$ decreases

absolute minimum of $U(\phi)$, then the $N+1$ particles are shifted radially away. The choice of this radial displacement is arbitrary. For example if $\mathbf{r}_{k}$ is transformed into $\mathbf{r}_{k}^{\prime},\left\|\mathbf{r}_{k}^{\prime}\right\|$ is defined as $\left\|\mathbf{r}_{k}\right\|+G$ (linear), or as $e^{G}\left\|\mathbf{r}_{k}\right\|$ (exponential). In both cases, $G$ is a parameter taking real positive values.

Iterating this process, one may reach a stationary regime: if $U(\phi)$ takes its absolute minimum at $\phi_{k}$ at time $k,\left(\phi_{k+1}-\phi_{k}\right) / 2 \pi(\bmod 1)$ may tend towards a fixed value $\theta$; in that case the particles form a spiral lattice. This asymptotic angle $\theta$ corresponds to the divergence characterizing a phyllotactic pattern. In their paper, Douady and Couder present a numerical plot of all possible values of $\theta$ versus $G$, for two interaction potentials. This plot looks entirely similar to the one obtained 
by Levitov, although the analysis of the last-mentioned can not apply to this case (disappearance of the symmetry).

The work of Douady and Couder contains the basic arguments to explain this hierarchical selection of noble numbers. In what follows we will prove some precise results concerning such situations, to which the analysis of Levitov can not apply on account of the absence of symmetry. Unlike what happens in the case of Levitov, the nature of the potential plays a crucial role: as the numerical counterexamples will show, noble numbers are selected insofar as a nearest neighbours approximation is valid. The tree structure of the spectrum then appears as the solution of a perturbed geometrical problem. This was implicitly included in the qualitative arguments given by Douady and Couder. A similar line of argument is found in earlier works (see for instance $[12,14])$.

1.3. The Divergence Spectrum. Both Levitov and Douady-Couder systems include a control parameter $G$ associated to the compression of the lattice. Such a parameter is common in models of phyllotaxis; Richards [12] introduced the term "plastochrone ratio" for the ratio of the distances of two successive leaves from the center of the botanical pattern. The divergence spectrum is the set of all possible divergences for every value of the control parameter. The explicative value of a model of phyllotaxis may be related to the properties of this spectrum; the distribution of divergences should notably present a peak around the golden mean and perhaps around other noble numbers. It is not easy to give a more precise criterion that is not controversial as far as biological realism is concerned. Let us say however that the tree structure of the spectrum of Levitov and Douady-Couder suggests a selection of the golden mean by an adiabatic decrease of $G$. Although this is a central idea in references $[8-10,14,18]$, we will not discuss it here but simply focus on the mathematical problem. A general study of the divergence spectra in models of phyllotaxis, including such questions, is made in reference [19,20].

As Guerreiro remarked $[19,20]$, there exists in some sense a formal equivalence between the problems of Levitov and Douady - Couder. This idea will be specified in what follows. Let us begin with the model of Douady and Couder. Suppose that the system has reached a stationary regime, characterized by a divergence $\theta$. If the particles are given an uniform radial speed, then they form a linear spiral lattice defined by the set:

$$
\operatorname{Sl}(G, \theta)=\left\{\mathbf{r}_{n}=(1+n G) \mathbf{I}_{2 \pi n \theta}, n \in \mathbb{N}\right\} .
$$

where

$$
\mathbf{I}_{\alpha}=\cos \alpha \cdot \mathbf{e}_{x}+\sin \alpha \cdot \mathbf{e}_{y} .
$$

Now this state is compatible with the dynamical rule defined in 1.2 if and only if the last particle is placed at the absolute minimum of the potential $U(\phi)$. This condition will be discussed in the limit of infinitely short-range interaction in Sect. 3.3. Let us consider here the weaker condition ${ }^{2}$ :

$$
\frac{d U}{d \phi}(0)=0
$$

\footnotetext{
2 Guerreiro $[19,20]$ studied the relationships between divergence spectra defined by different conditions ( 0 is the absolute minimum, local minimum, or extremum of $U$ ) in a very general framework. His analysis applies of course to our case.
} 
where

$$
U(\phi)=\sum_{n \geqq 1} u\left(\left\|\mathbf{I}_{\phi}-\mathbf{r}_{n}\right\|\right)
$$

and define the sequence of vectors

$$
\mathbf{R}_{n}=\mathbf{r}_{n}-\mathbf{r}_{0}, \quad n \geqq 1 .
$$

Equation (1.5) is equivalent to:

$$
\sum_{n \geqq 1} \frac{1}{\left\|\mathbf{R}_{n}\right\|} \frac{d u}{d r}\left(\left\|\mathbf{R}_{n}\right\|\right) \mathbf{R}_{n} \cdot \mathbf{e}_{y}=0 .
$$

From now on we will mainly be concerned with this last equation, which gives an implicit condition on $G$ and $\theta$. It is worthwhile to write it in a slightly different way. To that end we classify the vectors $\mathbf{R}_{n}$ according to increasing modulus; this defines a permutation $\sigma$ such that

$$
\left\|\mathbf{R}_{\sigma(n)}\right\| \leqq\left\|\mathbf{R}_{\sigma\left(n^{\prime}\right)}\right\| \text { if } n \leqq n^{\prime} .
$$

$\mathbf{R}_{\sigma(1)}$, for example, is the vector connecting $\mathbf{r}_{0}$ with its nearest neighbour in the spiral lattice. Let us also write:

$$
f(r)=-\frac{1}{r} \frac{d u}{d r}(r)
$$

Thus Eq. (1.8) takes the following form:

$$
H(G, \theta)=\sum_{n \geqq 1} f\left(\left\|\mathbf{R}_{\sigma(n)}(G, \theta)\right\|\right) \mathbf{R}_{\sigma(n)} \cdot \mathbf{e}_{y}=0 .
$$

Definition. The divergence spectrum is the set

$$
\Delta=\{(G, \theta): H(G, \theta)=0\}
$$

Consider Eq. (1.11); we can guess that if $f(r)$ decreases very sharply, the geometrical properties of the lattice $\operatorname{Sl}(G, \theta)$ will be determinant in the analysis of this equation. In fact a crucial role is played by the nearest neighbours of $\mathbf{r}_{0}$ whenever $H$ can be approximated in the following way:

$$
H(G, \theta) \cong \sum_{n=1}^{3} f\left(\left\|\mathbf{R}_{\sigma(n)}(G, \theta)\right\|\right) \mathbf{R}_{\sigma(n)} \cdot \mathbf{e}_{y}
$$

If $f(r)$ decreases abruptly enough, one has

$$
f\left(\left\|\mathbf{R}_{\sigma(1)}\right\|\right) \gg f\left(\left\|\mathbf{R}_{\sigma(2)}\right\|\right)
$$

and $h(g, \theta)$ can not cancel, unless

$$
\left\|\mathbf{R}_{\sigma(1)}\right\| \approx\left\|\mathbf{R}_{\sigma(2)}\right\|
$$

or

$$
\left|\mathbf{R}_{\sigma(1)} \cdot \mathbf{e}_{y}\right| \ll\left|\mathbf{R}_{\sigma(2)} \cdot \mathbf{e}_{y}\right| .
$$

These simple remarks lead to an analytical understanding of the results obtained by Douady and Couder. In fact it is already clear that whenever $f(r)$ decreases sharply enough, the divergence spectrum $\Delta$ will not be much different from the set defined 
by the geometrical conditions (1.15) and (1.16). Notice that (1.15) is analogous to the condition of tangency of circles discussed in Sect. 1.1. As we will see it defines a tree in the $(G, \theta)$ plane completely similar to the one of Fig. 2.

As regards the model of Levitov, the divergence spectrum is the set of couples

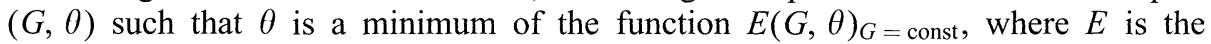
energy of a flux lattice (see 1.1)). Here we will simply describe the larger set $^{3}$

$$
\Delta^{\prime}=\left\{(G, \theta): H^{\prime}(G, \theta)=0\right\},
$$

where

$$
H^{\prime}(G, \theta)=\frac{\partial E}{\partial \theta}(G, \theta)
$$

or, more explicitly

$$
H^{\prime}=\sum_{m \in \mathbb{Z}} \sum_{n \geqq 1} \frac{1}{\left\|\mathbf{r}_{n m}\right\|} \frac{d u}{d r}\left(\left\|\mathbf{r}_{n m}\right\|\right) n \cdot \mathbf{r}_{n m} \cdot \mathbf{e}_{x} .
$$

In complete analogy with the previous case, let us classify the vectors $\mathbf{R}_{n m}=\mathbf{r}_{n m}-$ $\mathbf{r}_{00}=\mathbf{r}_{n m}$ according to increasing modulus. That defines two permutations, $\sigma$ and $\zeta$, such that

$$
\left\|\mathbf{R}_{\sigma(k) \zeta(k)}\right\| \leqq\left\|\mathbf{R}_{\sigma\left(k^{\prime}\right) \zeta\left(k^{\prime}\right)}\right\| \quad \text { if } k \leqq k^{\prime} .
$$

$\mathbf{R}_{\sigma(1) \zeta(1)}$ is thus the shortest vector in the lattice. For the sake of simplicity, we will simply write $\mathbf{R}_{\sigma(k)}$ instead of $\mathbf{R}_{\sigma(k) \zeta(k)}$ in what follows; it is to be inferred that the second index is always present. The formal analogy between $H$ and $H^{\prime}$ now becomes clear:

$$
H^{\prime}=\sum_{n \geqq 1} f\left(\left\|\mathbf{R}_{\sigma(n)}\right\|\right) \sigma(n) \cdot \mathbf{R}_{\sigma(n)} \cdot \mathbf{e}_{x} .
$$

The only significant difference with (1.11) lies in the fact that there is now an additional factor $\sigma(n)$; its presence does not require any fundamental change in the analysis. ${ }^{4}$ The same reasoning can therefore be followed and it leads to the same geometrical problem as in the case of Douady and Couder. Of course such an analysis is not only useless, since the question has been solved by Levitov, but it is unsuitable too, seeing that it is unaware of the symmetry of the problem. Nevertheless if one puts the problem in a different geometry, or if one removes the factor $\sigma(n)$ from (1.21), thus returning to Douady-Couder problem, the symmetry is destroyed. It is then necessary to go back to our analysis.

In the case of the periodic lattice, the geometrical problem has been solved and its solution possesses the Farey tree structure $[6,7]$. Our purpose here is to solve it in the case of a general class of spiral lattices (Sect. 2.3); as for the analysis of the spectrum $\Delta$, some results will be proved that are closely akin to the conclusions drawn by the above-mentioned authors on the basis of qualitative arguments (Sect. 3.1, 3.2,3.3). The effects of lattice compression and scale invariant interactions will be discussed. More precisely, if $u(r)$ is scale invariant $\left(u(r)=r^{-K}\right)$, it will be shown that if $K$ is great enough, there exists a continuous line in $\Delta$ connecting a rational divergence and the corresponding noble divergence on the axis $G=0$ (Sect. 3.2).

\footnotetext{
${ }^{3}$ In this case it is easy to deduce from $\Delta^{\prime}$ the spectrum defined by the minimality condition (just take into account the sign of $\frac{\partial E}{\partial \theta}(G, \theta)$ ).

${ }^{4}$ A change would be necessary if $\sigma(1) \ll \sigma(2)$ for example. This occurs only if a partial quotient in the continued fraction of $\theta$ is very large.
} 


\section{The Geometrical Problem}

2.1. The Set $\Sigma$. Consider a spiral lattice in the plane which may be linear (see (1.3)) or exponential:

$$
\operatorname{Se}(G, \theta)=\left\{\mathbf{r}_{n}=e^{G n} \mathbf{I}_{2 \pi n \theta}, n \in \mathbb{N}\right\}
$$

Imagine as well a periodic lattice on a cylinder, which is another classical representation of phyllotactic patterns. Unrolling the cylinder on the plane, one obtains the simple periodic lattice of Levitov:

$$
W(G, \theta)=\left\{\mathbf{r}_{n m}=(n \theta-m) \mathbf{e}_{x}+n G \mathbf{e}_{y}, n \in \mathbb{N} \text { and } m \in \mathbb{Z}\right\}
$$

For any of these lattices, we can define ${ }^{5}$ the permutation $\sigma$ and the sequence of vectors $\mathbf{R}_{\sigma(k)}, k \geqq 1$ (see (1.9) and (1.20)). Recall that in the case of $W(G, \theta)$, a second index $\zeta(k)$ is understood.

Now our problem is to describe the set

$$
\Sigma=\left\{(G, \theta):\left\|\mathbf{R}_{\sigma(1)}(G, \theta)\right\|=\left\|\mathbf{R}_{\sigma(2)}(G, \theta)\right\|\right\} .
$$

In other words, $\Sigma$ is the set of couples $(G, \theta)$ for which $\mathbf{r}_{0}$ has two equidistant nearest neighbours in the considered lattice. As was discussed in Sect. 1.3, $\Sigma$ is directly related to the divergence spectrum $\Delta$.

It is worth noting that in the case of $W(G, \theta)$ this problem is equivalent to the construction of lattices of tangent circles $[6,7]$; as a matter of fact, the properties of $W(G, \theta)$ are well known and it is useful to relate the study of spiral lattices to that of $W(G, \theta)$. This is best done through the following definition:

$$
\begin{gathered}
T_{\Phi}: \mathbb{R} / \mathbb{Z} \times \mathbb{R} \rightarrow \mathbb{R}^{2} \\
\left(\begin{array}{c}
x \\
y
\end{array}\right) \mapsto\left(\begin{array}{c}
\Phi(y) \cos 2 \pi x \\
\Phi(y) \sin 2 \pi x
\end{array}\right) .
\end{gathered}
$$

This transformation maps $W(1, \theta)$ on a spiral lattice $S_{\phi}(\theta)$ (see Fig. 4). If $\Phi(y)=$ $1+G y$, then $S_{\Phi}(\theta)=\operatorname{Sl}(G, \theta)$ and if $\Phi(y)=e^{G y}$, then $S_{\Phi}(\theta)=\operatorname{Se}(G, \theta)$. General conditions on $\Phi$ are discussed below.

2.2. The Farey Tree Structure of $\Sigma$ in the Case of the Simple Periodic Lattice. The set $\Sigma$ is completely described in references $[6,7]$ in the case of the periodic lattices $W(G, \theta)$. Its structure, that will be analysed in this section, is encountered in many other cases; we will therefore refer to the definitions introduced hereafter in the whole text. We have defined $\Sigma$ as:

$$
\Sigma=\left\{(G, \theta):\left\|\mathbf{R}_{\sigma(1)}(G, \theta)\right\|=\left\|\mathbf{R}_{\sigma(2)}(G, \theta)\right\|\right\}
$$

(see (2.3)). Here $G \in] 0,+\infty\left[\right.$ and $\theta \in[0,1 / 2]$. Recall that $\mathbf{R}_{\sigma(1)}$ and $\mathbf{R}_{\sigma(2)}$ are the shortest vectors in the lattice.

$\Sigma$ is a Cayley tree with branching number 3 . It can be described as an union of continuous lines (branches); every branch is of finite length and is connected to two other branches at each of its ends (see Fig. 5). Moreover there is a one-to-one

\footnotetext{
${ }^{5}$ Several definitions of $\sigma$ are of course possible whenever two vectors $\mathbf{R}_{n}$ have the same modulus.
} 


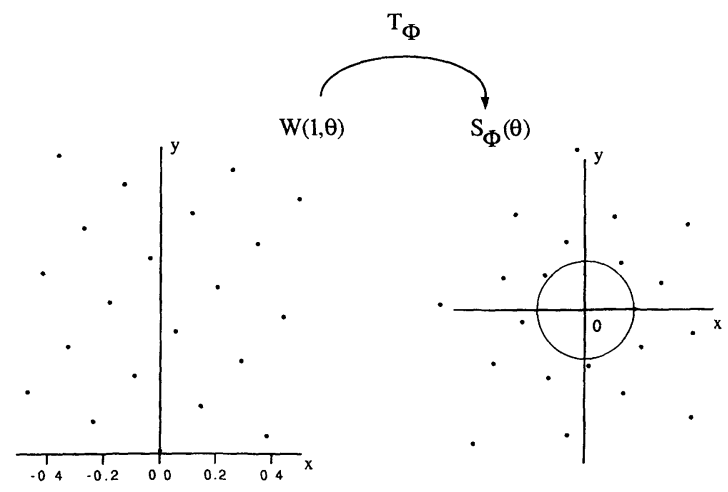

Fig. 4. The spiral lattice $S_{\Phi}(\theta)$ is the image of the periodic lattice $W(1, \theta)$ by the transformation $T_{\Phi}$

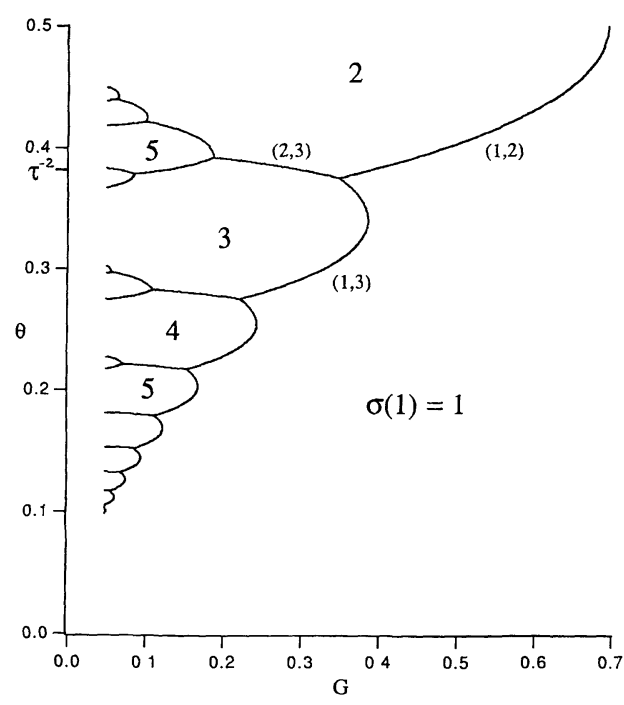

Fig. 5. The set $\Sigma$ for the family of spiral lattices $\operatorname{Se}(G, \theta)$. Notice that the regular branches $(1,2),(2,3),(3,5), \ldots$ lead to $\theta_{F}=\tau^{-2}=0.381966 \ldots$

correspondence between couples of coprime positive integers $(n, m)(n<m)$ and the set of branches, each branch $(n, m)$ being defined as:

$$
(n, m)=\left\{(G, \theta): \exists i, j \text { such that } \mathbf{r}_{n i}^{2}=\mathbf{r}_{m j}^{2} \leqq \mathbf{r}_{k l}^{2}, \forall k, 1\right\} .
$$

An $(n, m)$-branch is called regular if $2 n \geqq m$ and singular if $2 n<m$.

There is in fact a systematic way to construct $\Sigma$ : if one goes along a branch $(n, m)$ in the appropriate direction, one comes to a bifurcation point where $(n, m)$ is connected to $(n, n+m)$ and $(m, n+m) .(n, n+m)$ is a singular branch and $(m, n+m)$ is a regular branch (recall that $n<m)$. The type of a branch is easily determined since crossing a branch $(n, m)$ by decreasing $G$ always corresponds to a transition of first neighbour

$$
n \rightarrow m(n<m) .
$$


In other words, $\sigma(1)$ is always a decreasing function of $G$. This simple rule gives a universal structure to the bifurcation point (look at Fig. 5: there is always a bend if one follows the regular branch).

The set $\Sigma$ not only has this simple tree structure but it is closely related to number theory too. Suppose that $(G, \theta) \in(n, m)$ and write $\theta$ as a continued fraction

$$
\theta=\frac{1}{a_{1}+\frac{1}{a_{2}+\frac{1}{a_{3}+\ldots}}}=\left[a_{1}, a_{2}, a_{3}, \ldots\right] .
$$

Then there exists a positive integer $k$ such that

$$
n=q_{k}, \quad m=q_{k+1} \text { or } q_{k+2},
$$

where $q_{k}$ is the denominator of the $k^{\text {th }}$ principal convergent of $\theta$, that is

$$
\frac{p_{k}}{q_{k}}=\left[a_{1}, a_{2}, \ldots, a_{k}\right]
$$

An immediate consequence of this fact is that a branch $(n, m)$ never contains a point $\left(G, \frac{p}{q}\right)$, where $\frac{p}{q}$ is an irreducible fraction, with $q<m . \Sigma$ has indeed an important property: every singular branch $(n, m)$ contains a unique point $\left(G, \frac{p}{m}\right)$, where $\frac{p}{m}$ is an irreducible fraction, while a regular branch $\left(n^{\prime}, m\right)$ never contains such a point.

Definition. If a set $\Sigma$ has all the properties listed above, we say that it has the Farey tree structure.

Its construction is indeed equivalent to the construction of the Farey tree (cf. Fig. 3). The most interesting consequence of its properties is the following: let $(G, \theta) \in \Sigma$. There exists an unique path on $\Sigma$ connecting $(G=+\infty, \theta=1 / 2)$ to $(G, \theta)$. The continued fraction of $\theta$ is partly determined by the sequence of regular and singular branches constituting this path: $a_{i}-1$ is the number of singular branches preceding the $i^{\text {th }}$ regular branch in the path ${ }^{6}$. Thus if the path finishes with an infinite sequence of regular branches, it cuts the axis $G=0$ at a noble number $\theta$.

Further results and proofs can be found in references $[6,7]$. For an introduction to the Farey tree and continued fractions see references $[21,22]$.

Observe that the complementary set of $\Sigma$ in $] 0, \infty[\times[0,1 / 2]$ is a countable union of open sets; each of these sets is a connected domain, in which the function $\sigma(1)(G, \theta)$ is a constant. It is convenient to define the closure $\Sigma_{p / q}$ of such a domain, such that $(0, p / q) \in \Sigma_{p / q}$ (Fig. 6). A branch $(n, m)$ can thus be defined as the intersection of two sets $\Sigma_{\imath / n}$ and $\Sigma_{j / m}$.

2.3. The Farey Tree Structure of $\Sigma$ in the Case of the Spiral Lattices. The purpose of this section is to prove a theorem concerning the Farey tree structure of $\Sigma$ for a wide class of spiral lattices. The fact is that the distortion of the periodic lattices $W(G, \theta)$ into spiral lattices does not induce a deep change in the structure of $\Sigma$.

6 If we agree upon the following understanding: the first regular branch is $(1,2)$ if $\theta \in] 1 / 2,1[$ and $(n, n+1)(n \geqq 2)$ if $\theta \in] 0,1 / 2[$. 


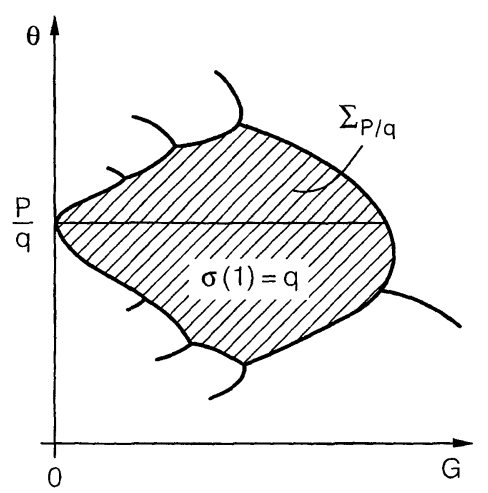

Fig. 6. Showing a set $\Sigma_{p / q}$. Any line $\theta=$ constant intersects at the most twice the boundary of $\Sigma_{p / q}$

We have defined in 2.1 the transformation

$$
\begin{gathered}
T_{\Phi}: \mathbb{R} / \mathbb{Z} \times \mathbb{R} \rightarrow \mathbb{R}^{2} \\
\left(\begin{array}{c}
x \\
y
\end{array}\right) \mapsto\left(\begin{array}{c}
\Phi(y) \cos 2 \pi x \\
\Phi(y) \sin 2 \pi x
\end{array}\right)
\end{gathered}
$$

which maps $W(1, \theta)$ on the spiral lattice $S_{\Phi}(\theta)$. Let us consider one-parameter families of differentiable functions $\Phi_{G}, G \in[0, \infty[$, which verify the general conditions:

$$
\begin{gathered}
\frac{d \Phi_{G}}{d x}(x)>0, \forall x \geqq 0 \text { and } \forall G>0, \\
\frac{d \Phi_{G}(x)}{d G}>0, \forall x \geqq 0 \text { and } \forall G \geqq 0, \\
\Phi_{G}(0)=1, \forall G \geqq 0, \\
\Phi_{0}(x)=1, \forall x \geqq 0, \\
\lim _{G \rightarrow+\infty} \Phi_{G}(1)=+\infty, \\
\lim _{x \rightarrow+\infty} \Phi_{G}(x)=+\infty, \forall G>0 .
\end{gathered}
$$

To each of these families of functions is associated a family of spiral lattices

$$
S_{\Phi_{G}}(\theta)=\left\{\mathbf{r}_{n}=\Phi_{G}(n) \mathbf{I}_{2 \pi n \theta}, n \in \mathbb{N}\right\} .
$$

These lattices are a simple generalization of the special cases $\operatorname{Sl}(G, \theta)$ and $\operatorname{Se}(G, \theta)$.

Theorem 1. Let $\Phi_{G}, G \in[0, \infty[$, be a one-parameter family of differentiable functions verifying the general conditions (2.9). Suppose further that:

$$
\begin{gathered}
\frac{d \log \Phi_{G}(n)}{d G}<\frac{d \log \Phi_{G}(m)}{d G}, \forall n<m, \forall G \geqq 0, \\
\frac{d^{2} \Phi_{G}}{d x^{2}}(x) \geqq 0, \forall x \geqq 0, \forall G>0 .
\end{gathered}
$$


Then the restriction of the set

$$
\Sigma=\left\{(G, \theta):\left\|\mathbf{R}_{\sigma(1)}(G, \theta)\right\|=\left\|\mathbf{R}_{\sigma(2)}(G, \theta)\right\|\right\}
$$

to the region $\left\|\mathbf{R}_{\sigma(1)}(G, \theta)\right\| \leqq 1$ has the Farey tree structure.

Before proving this theorem, let us mention an important corollary:

Corollary 2. The set $\Sigma$ related to the lattices $\operatorname{Sl}(G, \theta)$ and $\operatorname{Se}(G, \theta)$ has the Farey tree structure.

Proof of the corollary. Since $\left\|\mathbf{R}_{\sigma(1)}(G, \theta)\right\| \leqq 1$ if $G<0.05$ (see the remark after Proposition 6), it is enough to compute numerically the restriction of $\Sigma$ to the region $G>0.05$. In fact in this region $\sigma(1)$ can take only a finite number of values, so that a computer proof is possible.

Let us now come to a series of propositions that lead to the proof of Theorem 1 .

Proposition 3. $\sigma(1)(G, \theta)$ is the denominator of a principal convergent of $\theta$. It is true even in the degenerate case $\left\|\mathbf{R}_{\sigma(1)}\right\|=\left\|\mathbf{R}_{\sigma(2)}\right\|=\cdots=\left\|\mathbf{R}_{\sigma(m)}\right\|$ (that is, it is true for any choice of $\sigma(1))$.

Proof. Let $q_{r}$ be the greatest denominator of a principal convergent smaller than $\sigma(1)$. Suppose that $\sigma(1)$ itself is not the denominator of a principal convergent. This implies:

$$
\cos \left(2 \pi q_{r} \theta\right)>\cos (2 \pi \sigma(1) \theta)
$$

(it is a direct consequence of the properties of principal convergents). Thus

$$
\begin{aligned}
\mathbf{R}_{q_{r}}^{2}-\mathbf{R}_{\sigma(1)}^{2} & =\Phi^{2}\left(q_{r}\right)-\Phi^{2}(\sigma(1))+2\left(\Phi(\sigma(1)) \cos (2 \pi \sigma(1) \theta)-\Phi\left(q_{r}\right) \cos \left(2 \pi q_{r} \theta\right)\right) \\
& \leqq \Phi^{2}\left(q_{r}\right)-\Phi^{2}(\sigma(1))+2 \cos \left(2 \pi q_{r} \theta\right)\left(\Phi(\sigma(1))-\Phi\left(q_{r}\right)\right)<0, \quad(2.14)
\end{aligned}
$$

which is a contradiction.

The following lemma sums up some important facts concerning the lattice $W(1, \theta)$.

Lemma 4. Consider a lattice $W(1, \theta)$, and write $\theta$ as a continued fraction:

$$
\theta=\frac{1}{a_{1}+\frac{1}{a_{2}+\frac{1}{a_{3}+\ldots}}}=\left[a_{1}, a_{2}, a_{3}, \ldots\right] .
$$

The rational fractions

$$
\frac{p_{k, r}}{q_{k, r}}=: \frac{p_{k}+r p_{k+1}}{q_{k}+r q_{k+1}}, \quad r=1, \ldots, a_{k+2}-1
$$

are called the intermediate convergents of $\theta$. We construct two broken lines $P_{-}$ and $P_{+}$which are the convex envelopes of $W(1, \theta) \cap \mathbb{R}_{-} \times \mathbb{R}_{+}$and $W(1, \theta) \cap$ $\mathbb{R}_{+} \times \mathbb{R}_{+}$respectively. Now $P_{-}\left(P_{+}\right)$goes through points $\mathbf{r}_{q_{k} p_{k}}$ and $\mathbf{r}_{q_{k, r} p_{k, r}}$, where $r=1, \ldots, a_{k+2}-1$ and $k$ is odd(even).

Proof. See [23, 24].

\section{Definition.}

$$
\Omega_{t}=\left\{\mathbf{r} \in \mathbb{R}^{2}:\|\mathbf{r}\|>1 \text { and }\left\|\mathbf{r}-\mathbf{e}_{x}\right\|<t\right\} .
$$


Remark. It directly follows from this definition that $\sigma(1)=k$ and only if $\Omega_{\left|\mathbf{R}_{k}\right|} \cap$ $S_{\Phi}(\theta)=\varnothing$ or, equivalently, $T_{\Phi}^{-1}\left(\Omega_{\left|\mathbf{R}_{k}\right|}\right) \cap W(1, \theta)=\varnothing$. This brings us back to the study of $T_{\Phi}^{-1}\left(\Omega_{t}\right)$ and $W(1, \theta)$.

Significant properties of $S_{\Phi}(\theta)$ can be deduced from the hypothesis that $T_{\Phi}^{-1}\left(\Omega_{\left|\mathbf{R}_{\sigma(1)}\right|}\right)$ is a convex set.

Proposition 5. Consider a lattice $S_{\phi}(\theta)$; suppose that $T_{\Phi}^{-1}\left(\Omega_{\left|\mathbf{R}_{\sigma(1)}\right|}\right)$ is convex. Then $\left\|\mathbf{R}_{\sigma(4)}\right\|>\left\|\mathbf{R}_{\sigma(1)}\right\|$. Moreover if $\left\|\mathbf{R}_{\sigma(1)}\right\|=\left\|\mathbf{R}_{\sigma(2)}\right\|=\left\|\mathbf{R}_{\sigma(3)}\right\|$, then $\{\sigma(1), \sigma(2)$, $\sigma(3)\}=\left\{q_{k}, q_{k+1}, q_{k+2}\right\}$, where $q_{k}, q_{k+1}, q_{k+2}$ are the denominators of three successive principal convergents of $\theta$, and $a_{k+2}=1$.

Proof. Suppose that $\left\|\mathbf{R}_{\sigma(1)}\right\|=\left\|\mathbf{R}_{\sigma(2)}\right\|=\left\|\mathbf{R}_{\sigma(3)}\right\|$; this amounts to saying that $T_{\Phi}^{-1}\left(\mathbf{R}_{\sigma(1)}\right), T_{\Phi}^{-1}\left(\mathbf{R}_{\sigma(2)}\right)$ and $T_{\Phi}^{-1}\left(\mathbf{R}_{\sigma(3)}\right)$ all lie on the boundary of $T_{\Phi}^{-1}\left(\Omega_{\left|\mathbf{R}_{\sigma(1)}\right|}\right)$. By Proposition 3 and Lemma 4, these points also belong to the convex envelopes $P_{-}$and $P_{+}$. Now suppose that two of them belong to $P_{-}$. Because of the convexity of $T_{\Phi}^{-1}\left(\Omega_{\left|\mathbf{R}_{\sigma(1)}\right|}\right)$ and $P_{-}$, these must be two successive points of $P_{-}$(otherwise some lattice point would lie inside $T_{\Phi}^{-1}\left(\Omega_{\left|\mathbf{R}_{\sigma(1)}\right|}\right)$ ). They correspond therefore to two successive denomiators $q_{k}, q_{k+2}$ with $a_{k+2}=1$. A similar reasoning shows that the third point lies on $P_{+}$. Let us prove that it corresponds to $q_{k+1}$. Since $\mathbf{r}_{q_{k} p_{k}}, \mathbf{r}_{q_{k+2} p_{k+2}}, \mathbf{r}_{q_{k+1,1} p_{k+1,1}}$ are three aligned points,only two can lie on the boundary of a convex set: thus $\mathbf{r}_{q_{k+1,1} p_{k+1,1}}$ (and any point of $P_{+}$above it) is strictly outside of $T_{\Phi}^{-1}\left(\Omega_{\left|\mathbf{R}_{\sigma(1)}\right|}\right)$. A similar reasoning shows that $\mathbf{r}_{q_{k-1, a_{k+1}-1} p_{k-1, a_{k+1}-1}}$ (and any point of $P_{+}$below it) is also situated strictly outside of $T_{\Phi}^{-1}\left(\Omega_{\left|\mathbf{R}_{\sigma(1)}\right|}\right)$. Consequently the only point of $P_{+}$that can be on the boundary of $T_{\Phi}^{-1}\left(\Omega_{\left|\mathbf{R}_{\sigma(1)}\right|}\right)$ is $\mathbf{r}_{q_{k+1}} p_{k+1}$.

Proposition 6. Suppose that $\frac{d^{2} \Phi}{d x^{2}}(x) \geqq 0, \forall x \geqq 0$. If $t \leqq 1, T_{\Phi}^{-1}\left(\Omega_{t}\right)$ is a convex set.

The proof of this proposition is given in Appendix A.

Remark. It is obvious that $\left\|\mathbf{R}_{\sigma(1)}(G, \theta)\right\|$ tends towards zero in the limit $G \rightarrow 0$. It is nevertheless useful to have an estimate of $\left\|\mathbf{R}_{\sigma(1)}\right\|$ that is independent of $\theta$.

If $G \leqq G_{0}$,where $\Phi_{G_{0}}(7)=\sqrt{2}$, then $\left\|\mathbf{R}_{\sigma(1)}(G, \theta)\right\| \leqq 1, \forall \theta \in[0,1 / 2]$. To prove this inequality, first notice that $\left\|\mathbf{R}_{k}(G, \theta)\right\| \leqq 1$ if and only if

$$
\frac{\Phi_{G}(k)}{2 \cos (2 \pi k \theta)} \leqq 1
$$

One has therefore to prove

$$
\min _{k>1} \frac{\Phi_{G}(k)}{2 \cos (2 \pi k \theta)} \leqq 1, \forall \theta \in[0,1 / 2], \forall G \leqq G_{0} .
$$

Now a stronger inequality holds

$$
\max _{1 \leqq k \leqq 7} \cos (2 \pi k \theta) \geqq \frac{1}{\sqrt{2}}, \forall \theta \in[0,1] .
$$

It is necessary to be more specific about $\Phi_{G}$ in order to describe the set $\Sigma$. 
Proposition 7. Suppose that

$$
\frac{d \log \Phi_{G}(n)}{d G}<\frac{d \log \Phi_{G}(m)}{d G}, \forall 1 \leqq n<m, \forall G \geqq 0 .
$$

Consider the function

$$
A_{n m}(G, \theta)=\mathbf{R}_{n}^{2}(G, \theta)-\mathbf{R}_{m}^{2}(G, \theta), n<m .
$$

For any fixed value of $\theta$ it has at the most one zero $G_{0}$; it is negative for $G>G_{0}$ and positive otherwise.

Corollary 8. Suppose that

$$
\frac{d \log \Phi_{G}(n)}{d G}<\frac{d \log \Phi_{G}(m)}{d G}, \forall 1 \leqq n<m, \forall G \geqq 0 .
$$

Consider the function $\sigma(1)(G, \theta)$ defined on $\left(\mathbb{R}_{+} \times[0,1 / 2]\right) \backslash \Sigma$. It is a decreasing function of $G$. Moreover $\sigma(1)$ cannot be constant in an open neighbourhood of a point of $\Sigma$.

The proof of Proposition 7 is given in Appendix A.

Remark. One may point out that

$$
\frac{d \log \Phi_{G}(n)}{d G}<\frac{d \log \Phi_{G}(m)}{d G}, \forall 1 \leqq n<m, \forall G \geqq 0
$$

if and only if

$$
\frac{d}{d G}\left(\frac{\Phi_{G}(n)}{\Phi_{G}(m)}\right)<0, \forall 1 \leqq n<m, \forall G \geqq 0 .
$$

It implies that

$$
\Phi_{G}(m)-\Phi_{G}(n)
$$

is an increasing function of $G$. The reciprocal is not true.

Proposition 9. Suppose that

$$
\frac{d \log \Phi_{G}(n)}{d G}<\frac{d \log \Phi_{G}(m)}{d G}, \forall 1 \leqq n<m, \forall G \geqq 0 .
$$

Let us define the set $\Sigma\left(G_{0}\right)$ :

$$
\Sigma\left(G_{0}\right)=\left\{(G, \theta):(G, \theta) \in \Sigma, G>G_{0}\right\} .
$$

For any $G_{0}>0, \Sigma\left(G_{0}\right)$ is connected. It is a finite union of continuous lines in the $(G, \theta)$ plane, each line being an implicit solution of:

$$
\mathbf{R}_{n}^{2}(G, \theta)-\mathbf{R}_{m}^{2}(G, \theta)=0
$$

for given values of $n$ and $m$.

Let us call these lines $(n, m)$-branches (we always assume $n<m$ ).

A branch is connected to two other branches at each of its ends (or more, but this is not generic), and it is generically not connected elsewhere to any branch. 
The (1,2)-branch is the only branch that crosses the symmetry axis $\theta=1 / 2$. If $\left(G^{*}, 1 / 2\right)$ is the intersection point, then $G^{*}=\max _{(G, \theta) \in \Sigma} G$.

Proof. It is shown in the proof of Proposition 7 that the implicit solutions of (2.22) have no singular points. Furthermore it is clear that for any $G_{0}>0, \Sigma\left(G_{0}\right)$ is a finite union of such solutions, since

$$
\lim _{x \rightarrow+\infty} \Phi_{G}(x)=+\infty, \forall G>0 .
$$

It is easy to prove now that $\Sigma\left(G_{0}\right)$ is connected. It is impossible indeed to construct a continuous path in $] 0, \infty[\times \mathbb{R}$ that encloses a point of $\Sigma$ and does not intersect $\Sigma$ (see Corollary 8 ). It is also impossible to construct a continuous path in $] 0, \infty[\times \mathbb{R}$ that connects two points on the axis $G=0$ and does not intersect $\Sigma$. To see this observe that the function $\sigma(1)(G, \theta)$ is constant along such a path. Suppose $\sigma(1)(G, \theta)=q$. The path must then cut the axis $G=0$ at two rational values $\theta=p / q$ and $\theta=p^{\prime} / q$. Now there exists a rational number $p^{\prime \prime} / q^{\prime}, q^{\prime}<q$, such that $p / q<p^{\prime \prime} / q^{\prime}<p^{\prime} / q$. This contradicts Corollary 8 since $\sigma(1)\left(G, \theta=p^{\prime \prime} / q^{\prime}\right)=q^{\prime}<q$ if $G$ is small enough.

Generically a branch is connected to two other branches at each of its ends, and it is not connected elsewhere to any branch. To prove this, let us examine all the other possibilities.

A $(n, m)$-branch can not end at $G=0$. For if this branch contains the point $(G=0, \theta=\tilde{\theta})$, then $\cos (2 \pi n \tilde{\theta})=\cos (2 \pi m \tilde{\theta})=1$, so that $\tilde{\theta}=p / q$ and $q|n, q| m$. Therefore $m=k q$, where $k \geqq 2$. Now $\forall k \geqq 2$ there exists $\varepsilon$ such that $\forall G>0, \forall|\theta-p / q|<\varepsilon,\left\|\mathbf{R}_{k q}(G, \theta)\right\|>\left\|\mathbf{R}_{q}(G, \theta)\right\|$. This is a contradiction.

A branch can neither cross the axis $\theta=0(\bmod 1)$ nor continue up to $G=+\infty$. This is easily deduced from the following inequalities:

$$
\begin{gathered}
\left\|\mathbf{R}_{\sigma(1)}(G, 0)\right\|=\Phi_{G}(1)-1>\Phi_{G}(2)-1=\left\|\mathbf{R}_{\sigma(2)}(G, 0)\right\|, \forall G>0, \\
\frac{\mathbf{R}_{k}^{2}(G, \theta)-\mathbf{R}_{1}^{2}(G, \theta)}{\Phi_{G}^{2}(k)} \geqq 1-\frac{\Phi_{G}^{2}(1)}{\Phi_{G}^{2}(k)}-\frac{4}{\Phi_{G}(k)}, \forall k>1 .
\end{gathered}
$$

The right-hand side of Eq. (2.25) is strictly positive when $G$ is great enough, since $\frac{\Phi_{G}^{2}(1)}{\Phi_{G}^{2}(k)}$ is a decreasing function of $G$.

Now we have proved in Proposition 7 that a branch can be described as the plot of a continuous function $G(\theta)$; the discussion above shows that this function is defined on a closed interval in ]0,1 [ and it has a finite upper bound and a positive lower bound. That amounts to saying that an $(n, m)$-branch has two ends in the $(G, \theta)$ plane.

It is impossible that a branch has an isolated end. If it were so, one could construct a path enclosing this end and intersecting $\Sigma$ at one point only. This would contradict Corollary 8.

Therefore a branch is necessarily connected to one or two other branches at each of its ends (if it were connected to more than two branches, there would be four equidistant nearest neighbours of the origin in the lattice; this is not generic). Now if it were connected to one branch only, one could construct a closed path cutting both branches and intersecting $\Sigma$ at two points only. This is impossible (see Corollary 8). 
Suppose that a branch is connected to another one at a point not coinciding with an end; these branches must be tangent to each other, unless there are four nearest neighbours of the origin in the lattice. This is obviously not generic.

Finally it is not difficult to see that the axis $\theta=1 / 2$ intersect the set $\Sigma$ at a point $\left(G^{*}, 1 / 2\right)$ belonging to a $(1,2)$-branch. If $G>G^{*}$, then $(G, \theta) \notin \Sigma, \forall \theta \in[0,1]$, since $\forall k \geqq 2, \forall G>G^{*}$,

$$
\left\|\mathbf{R}_{k}\right\|-\left\|\mathbf{R}_{1}\right\| \geqq \Phi_{G}(k)-\Phi_{G}(1)-2 \geqq \Phi_{G}(2)-\Phi_{G}(1)-2>0 .
$$

\section{Proposition 10. Suppose that}

$$
\frac{d \log \Phi_{G}(n)}{d G}<\frac{d \log \Phi_{G}(m)}{d G}, \forall 1 \leqq n<m, \forall G \geqq 0 .
$$

$A$ regular $(n, m)$-branch contains no point of type $(G, p / m), p \in \mathbb{N}$.

A singular ( $n, m)$-branch that is connected to an $(n, n+m)$ and an $(m, n+m)$ branch at one end, and to an $(n, m-n)$ and an $(m-n, m)$-branch at the other end, contains a point of type $(G, p / m), p \in \mathbb{N}$.

Proof. If $2 n \geqq m$ it is not difficult to verify that

$$
\left\|\mathbf{R}_{m-n}(G, \theta=p / m)\right\|<\left\|\mathbf{R}_{n}(G, \theta=p / m)\right\|, \forall G>0,
$$

thus a regular $(n, m)$-branch can not contain a point $(G, p / m), p \in \mathbb{N}$.

Suppose that $2 n<m$ and that $(n, m)$ is connected to $(n, n+m)$ and $(m, n+m)$ at one end, and to $(n, m-n)$ and $(m-n, m)$ at the other end. We want to prove that $\mathbf{R}_{m} \cdot \mathbf{e}_{y}$ does not have a constant sign along the $(n, m)$-branch. Clearly $\mathbf{R}_{n} \cdot \mathbf{e}_{y}$ can not change its sign along $(n, m)$. Now $\mathbf{R}_{n} \cdot \mathbf{e}_{y}$ and $\mathbf{R}_{m} \cdot \mathbf{e}_{y}$ have opposite signs at the bifurcation point $\left\|\mathbf{R}_{n}\right\|=\left\|\mathbf{R}_{m}\right\|=\left\|\mathbf{R}_{n+m}\right\|$ (otherwise $n+m$ would not be the denominator of a convergent) and they have the same sign at the bifurcation point $\left\|\mathbf{R}_{n}\right\|=\left\|\mathbf{R}_{m-n}\right\|=\left\|\mathbf{R}_{m}\right\|$ (otherwise $\left\|\mathbf{R}_{m-n}\right\|>\left\|\mathbf{R}_{n}\right\|$ ).

Let us proceed to the proof of Theorem 1. It is composed of several steps.

1) In the region $\left\{(G, \theta): \theta \in[0,1 / 2],\left\|\mathbf{R}_{\sigma(1)}(G, \theta)\right\| \leqq 1\right\}$, there is at the most one point $\left(G_{0}, \theta_{0}\right)$ such that $\left\|\mathbf{R}_{\sigma(1)}\left(G_{0}, \theta_{0}\right)\right\|=\left\|\mathbf{R}_{q_{k}}\right\|=\left\|\overline{\mathbf{R}}_{q_{k+1}}\right\|=\left\|\mathbf{R}_{q_{k+2}}\right\|$, for any fixed values of $q_{k}, q_{k+1}, q_{k+2}$. Suppose in fact that there exists two such points $\left(G_{0}, \theta_{0}\right)$ and $\left(G_{0}^{\prime}, \theta_{0}^{\prime}\right), G_{0}^{\prime}<G_{0}, \theta_{0}^{\prime}<\theta_{0}$. It follows from Proposition 7 that at $\left(G_{0}^{\prime}, \theta_{0}\right)$ :

$$
\left\|\mathbf{R}_{q_{k}}\right\|>\left\|\mathbf{R}_{q_{k+1}}\right\|>\left\|\mathbf{R}_{q_{k+2}}\right\| \text {. }
$$

It is quite clear that $\cos \left(2 \pi q_{k+1} \theta\right)$ and $\cos \left(2 \pi q_{k+2} \theta\right)$ can not cancel in $\left[\theta_{0}^{\prime}, \theta_{0}\right]$, since $q_{k}, q_{k+1}, q_{k+2}$ must be denominators of convergents of $\theta_{0}$ and $\theta_{0}^{\prime}$ (cf. Prop. 5). This implies that either $\left\|\mathbf{R}_{q_{k+1}}\right\|-\left\|\mathbf{R}_{q_{k+2}}\right\|$ or $\left\|\mathbf{R}_{q_{k}}\right\|-\left\|\mathbf{R}_{q_{k+1}}\right\|$ increases from $\left(G_{0}^{\prime}, \theta_{0}\right)$ to $\left(G_{0}^{\prime}, \theta_{0}^{\prime}\right)$. This is of course impossible.

2) In the region $\left\{(G, \theta): \theta \in[0,1],\left\|\mathbf{R}_{\sigma(1)}(G, \theta)\right\| \leqq 1\right\}$ an $(n, m)$-branch is connected to an $(n, n+m)$ and an $(m, n+m)$-branch at one end, and to an $(m-n, n)$ and an $(m-n, m)$ - branch at the other end. It is not connected elsewhere to any branch. Each $(n, m)$-branch is unique in this region.

This is a direct consequence of 1) and Propositions 5 and 9.

3) Consider an $(n, m)$-branch whose ends both belong to the region $\left\|\mathbf{R}_{\sigma(1)}\right\| \leqq 1$. If it is a regular branch, that is $2 n \geqq m$, it contains no point of type $(G, p / m)$, $p \in \mathbb{N}$. If it is a singular branch, that is $2 n<m$, it always contains such a point.

This is a direct consequence of 2) and Proposition 10. 
4) The restriction of $\Sigma$ to the region $\left\|\mathbf{R}_{\sigma(1)}\right\| \leqq 1$ is the part of a Cayley tree with branching number 3 . It is in fact impossible to construct a simple closed path in this restriction of $\Sigma$.

This follows from 2 .

This completes the proof of the theorem.

\section{An Analysis of the Divergence Spectrum}

3.1. Short Range Interactions and Noble Numbers Selection Rule. We want to show in this paragraph that a sharp decrease of $f(r)$ (see (1.10)) induces a hierarchical selection of noble numbers in the spectrum $\Delta$. An explicit family of functions is considered here

$$
f_{K}(r)=e^{-K r}, K \in R_{+}^{*},
$$

but the results are easily extended to other profiles. The important thing is that $f_{K}(r)$ decreases exponentially with a characteristic length $1 / K$.

Our aim is to describe the set

$$
\Delta_{K}=\left\{(G, \theta): H_{K}(G, \theta)=0\right\},
$$

where (cf. (1.11) and (1.12))

$$
H_{K}(G, \theta)=\sum_{n \geqq 1} f_{K}\left(\left\|\mathbf{R}_{\sigma(n)}(G, \theta)\right\|\right) \mathbf{R}_{\sigma(n)} \cdot \mathbf{e}_{y}=0
$$

in the case of linear or exponential spiral lattices, or any lattice family such that $\Sigma$ has the Farey tree structure. Some additional definitions are necessary. Consider the set

$$
\Sigma^{*}=\Sigma \cup\left\{(G, \theta): \mathbf{R}_{\sigma(1)}(G, \theta) \cdot \mathbf{e}_{y}=0\right\} .
$$

Since $\Sigma$ has the Farey tree structure, $\Sigma^{*}$ is simply obtained by drawing segments $\theta=\frac{p}{m}$ connected to singular branches $(n, m)$ (Fig. 7). These rational divergences will play an important role in the following; their relevance to the geometrical or

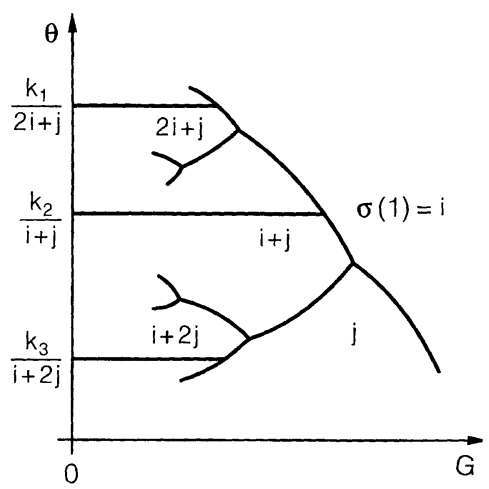

Fig. 7. $\Sigma^{*}$ is obtained by constructing the intersection of the line $\theta=p / q$ with the set $\Sigma_{p / q}, \forall p / q \in$ $\mathbb{Q} \cap[0,1 / 2]$ 
dynamical problem was pointed out by many authors (see e.g. $[5,15,19,20]$ ). Let us construct successive approximations of $\Sigma^{*}$ :

$$
\Sigma^{*}(\varepsilon)=\left\{(G, \theta): \exists \delta \text { such that }|\delta|<\varepsilon \text { and }(G, \theta+\delta) \in \Sigma^{*}\right\}, \varepsilon>0 .
$$

This allows us to give a precise statement about $\Delta_{K}$ in the limit $K \rightarrow+\infty$.

Theorem 11. $\forall \varepsilon>0, \exists K_{0}$ such that for any $K>K_{0}$,

$$
\Delta_{K} \subset \Sigma^{*}(\varepsilon) \text {. }
$$

Proof. We consider here the general case of a lattice family given by a oneparameter family of functions $\Phi_{G}, G \in[0, \infty[$. To each function is associated a spiral lattice

$$
S_{\Phi_{G}}(\theta)=\left\{\mathbf{r}_{n}=\Phi_{G}(n) \mathbf{I}_{2 \pi n \theta}, n \in \mathbb{N}\right\} .
$$

Some general conditions on $\Phi_{G}$ are listed in Sect. 2.3. Here we also assume that

$$
\frac{d^{2} \Phi_{G}}{d^{2} x} \geqq 0
$$

so that

$$
\sum_{n \geqq 2} \Phi_{G}(n) e^{-K\left(\Phi_{G}(n)-\Phi_{G}(1)\right)} \leqq \sum_{n \geqq 2} \Phi_{G}(n) e^{-K \Phi_{G}(n) / 2} .
$$

Let us define the set $\Lambda=] 0,+\infty\left[\times[0,1] \backslash \Sigma^{*}(\varepsilon)\right.$. If $K$ is great enough, then

$$
\inf _{(G, 0) \in \Lambda}\left|\mathbf{R}_{\sigma(1)}(G, \theta) \cdot \mathbf{e}_{y}\right|>\sup _{(G, \theta) \in \Lambda}\left|\sum_{i \geqq 2} \frac{f_{K}\left(R_{\sigma(i)}\right)}{f_{K}\left(R_{\sigma(1)}\right)} \mathbf{R}_{\sigma(i)}(G, \theta) \cdot \mathbf{e}_{y}\right| .
$$

The left-hand term is indeed strictly positive and independent of $K$, and the righthand term tends towards zero as $K$ tends towards infinity. This follows from (3.4) and from the fact that

$$
\inf _{(G, \theta) \in \Lambda}\left(\left\|\mathbf{R}_{\sigma(2)}(G, \theta)\right\|-\left\|\mathbf{R}_{\sigma(1)}(G, \theta)\right\|\right)>0 .
$$

This proves the theorem. Notice that (3.6) holds because $\Lambda \cap] 0, G] \times[0,1]$ is a compact set (the different branches of $\Sigma^{*}(\varepsilon)$ must overlap).

Theorem 11 shows the connection between $\Delta_{K}$ and $\Sigma$. As for Theorem 12, it can be seen as a consequence of the essential difference between $\Delta_{K}$ and $\Sigma$, that is the existence of an infinity of bifurcation points in $\Sigma$ and their absence in $\Delta_{K}$. Near each bifurcation point of $\Sigma$ we will construct a line $\Gamma$ that crosses the singular branch and divides $\Sigma^{*}(\varepsilon)$ in two parts (see Fig. 8). The theorem says that no point of $\Delta_{K}$ lies on $\Gamma$.

Theorem 12. Let $K_{0}$ be such that $\Delta_{K} \subset \Sigma^{*}(\varepsilon)$ if $K>K_{0}$. Let $(j, q)$ be a singular branch of $\Sigma$. One constructs the broken line $\Gamma$ illustrated on Fig. 8: $\Gamma$ is included in $\Sigma^{*}(\varepsilon)$, crosses $(j, q)$ and divides $\Sigma^{*}(\varepsilon)$ in two parts. If $\varepsilon$ is small enough and if $K>K_{0}$ is great enough then

$$
\Delta_{K} \cap \Gamma=\varnothing \text {. }
$$

Proof. Let $\left\{\left(G_{0}, p / q\right)\right\}=\Sigma \cap \Gamma$. One has:

$$
\left(\left\|\mathbf{R}_{\sigma(3)}\right\|-\left\|\mathbf{R}_{\sigma(2)}\right\|\right)\left(G_{0}, p / q\right)>0,
$$




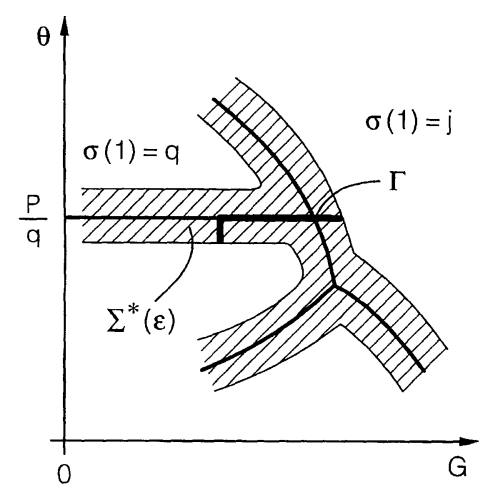

Fig. 8. $\Gamma$ is the union of two perpendicular segments one of which is parallel to the line $\theta=p / q$. The length of $\Gamma$ can be made as small as possible

so that if $\varepsilon$ is small enough

$$
\inf _{(G, \theta) \in \Gamma}\left(\left\|\mathbf{R}_{\sigma(3)}\right\|-\left\|\mathbf{R}_{\sigma(2)}\right\|\right)(G, \theta)=C>0 .
$$

Let us prove that if $K$ is great enough,

$$
\begin{gathered}
\inf _{(G, \theta) \in \Gamma}\left|\frac{f_{K}\left(R_{\sigma(1)}\right)}{f_{K}\left(R_{\sigma(2)}\right)} \mathbf{R}_{\sigma(1)}(G, \theta) \cdot \mathbf{e}_{y}+\mathbf{R}_{\sigma(2)}(G, \theta) \cdot \mathbf{e}_{y}\right|> \\
\sup _{(G, \theta) \in \Gamma}\left|\sum_{i \geqq 3} \frac{f_{K}\left(R_{\sigma(i)}\right)}{f_{K}\left(R_{\sigma(2)}\right)} \mathbf{R}_{\sigma(i)}(G, \theta) \cdot \mathbf{e}_{y}\right| .
\end{gathered}
$$

The right-hand term tends towards zero as $K$ tends towards infinity. Now the lefthand term is always greater than $\sin (2 \pi / q)$. To see this observe that if $(G, \theta) \in \Gamma$, then $\{\sigma(1), \sigma(2)\}=\{j, q\}$. Moreover $\mathbf{R}_{q} \cdot \mathbf{e}_{y}$ and $\mathbf{R}_{j} \cdot \mathbf{e}_{y}$ have the same sign, and $\left|\mathbf{R}_{J} \cdot \mathbf{e}_{y}\right| \geqq \sin (2 \pi / q)$.

By definition $\Delta_{K}$ is an union of continuous lines (implicit functions). Theorems 11 and 12 show that if $K$ is great enough, each line must be contained in a set of tongues of width $\varepsilon^{7}$. Each tongue follows a half-loop that goes from $(G=0, \theta=$ $p / q)$ to a singular branch $(j, q)$ and then along successive regular branches of $\Sigma$ (Fig. 9). In other words, a half-loop connects two points on the $G=0$ axis; one is a point with a rational ordinate $\theta=\left[a_{1}, \ldots, a_{n}\right]\left(a_{n}>1\right)$ and the other is a point whose ordinate is a noble number $\theta=\left[a_{1}, \ldots, a_{n}, 1,1,1, \ldots\right]$. Of course these tongues overlap when $G$ is too small, so that noble numbers are approached with an accuracy of order $\varepsilon$ (the accuracy is necessarily finite because of the characteristic length of the interaction).

This situation is illustrated in Fig. 10; the structure of this spectrum can be directly compared with the solution of the geometrical problem (Fig. 5).

Fig. 11 shows how this structure disappears when $K$ is too small. This contrasts with the special case considered by Levitov: the spectrum is not modified by the range of the interaction (Fig. 12).

\footnotetext{
7 Conversely, there must be a line in most tongues: in the limit $K \rightarrow+\infty, G \rightarrow 0, H_{K}(G, \theta)$ must change its sign around $\theta=p / q$. We will return to this point in section 3.2 .
} 


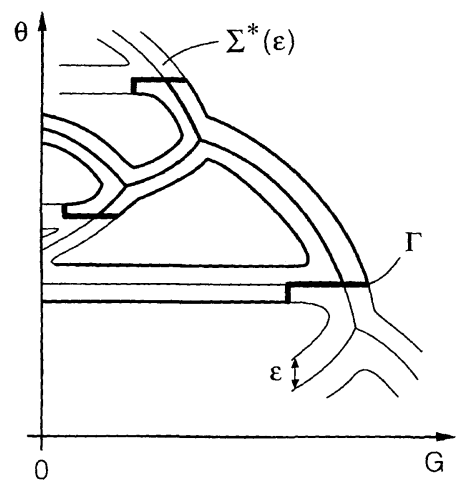

Fig. 9. A continuous line of $\Delta$ can not follow the singular branches of $\Sigma$ that are crossed by a broken line $\Gamma$

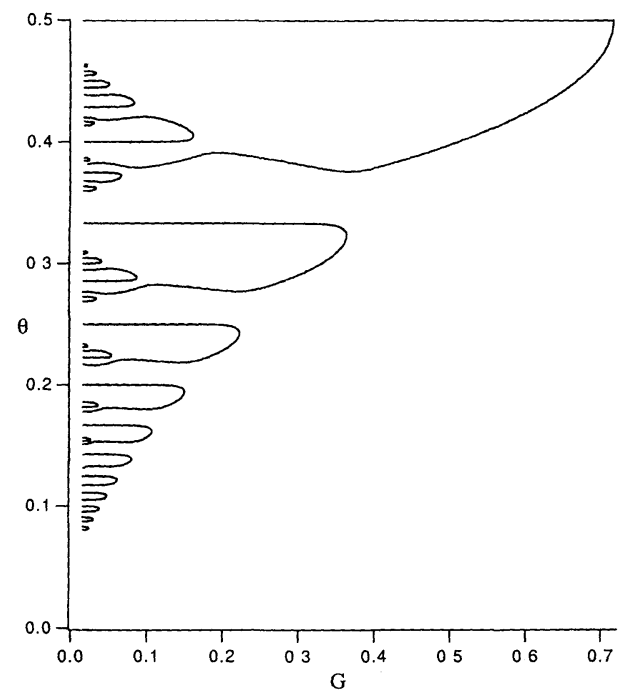

Fig. 10. The divergence spectrum $\Delta$ with the condition of Douady-Couder. The family of spiral lattices is $\operatorname{Se}(G, \theta)$ and $-\frac{1}{r} \frac{d u}{d r}=e^{-K r}, K=10$

If $K$ is large enough, the structure of the spectrum remains essentially unchanged if one considers the periodic lattice instead of a spiral lattice, or the variational problem of Levitov instead of the condition of Douady-Couder (see Fig. 13).

3.2. Scale Invariant Interactions. If the potential $u$ is a power law, that is

$$
f_{K}(r)=r^{-K}
$$

the question of whether noble numbers are exactly selected naturally comes to mind. In this case in fact there is no characteristic length of the potential, so that the nearest neighbours approximation (1.13) may be valid for all values of $G$. 


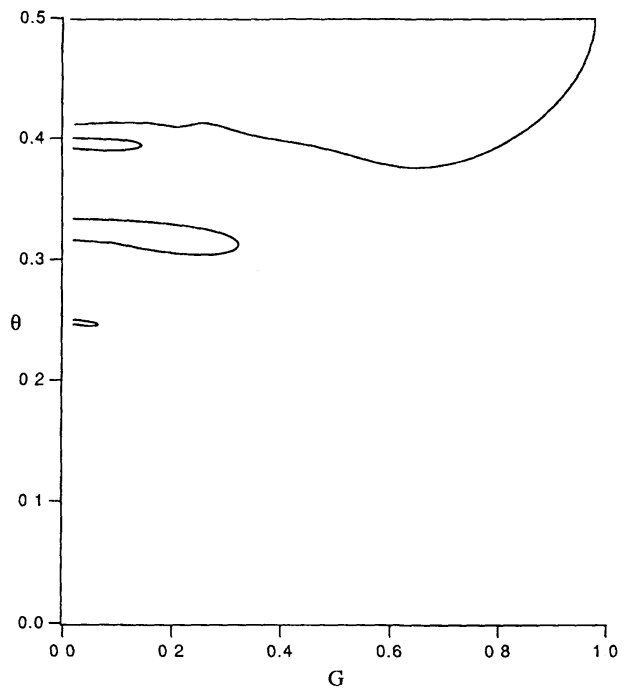

Fig. 11. The spectrum $\Delta$ with the condition of Douady-Couder. The family of spiral lattices is $\operatorname{Se}(G, \theta)$ and $-\frac{1}{r} \frac{d u}{d r}=e^{-K r}, K=0.7$

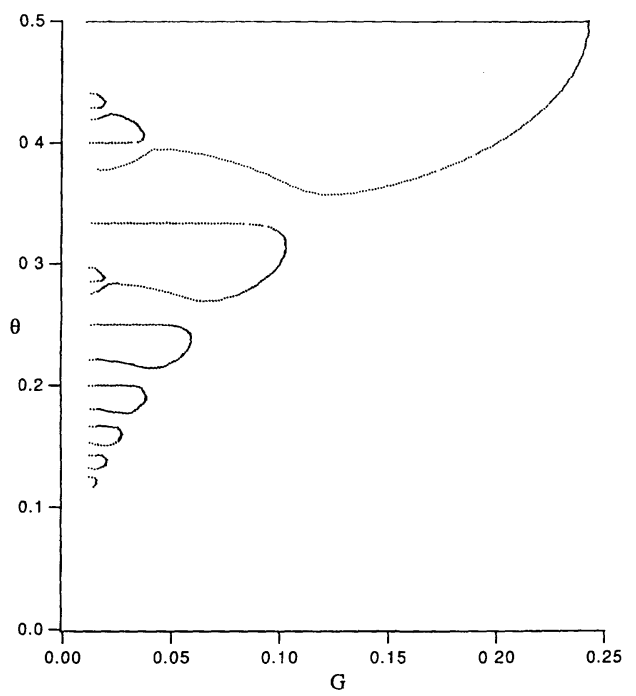

Fig. 12. The spectrum $\Delta$ with the condition of Levitov. The family of periodic lattices is $W(G, \theta)$ and $-\frac{1}{r} \frac{d u}{d r}=e^{-K r}, K=0.7$

The following discussion shows that the answer to this question is positive; nevertheless all noble numbers can not be considered equivalent.

The first step in the analysis is the construction of the set $\Sigma^{*}(\alpha, \beta)$, such that $H_{K}(G, \theta)$ can not cancel outside of $\Sigma^{*}(\alpha, \beta)$. The rest of the argument closely follows the reasoning of the previous section. 


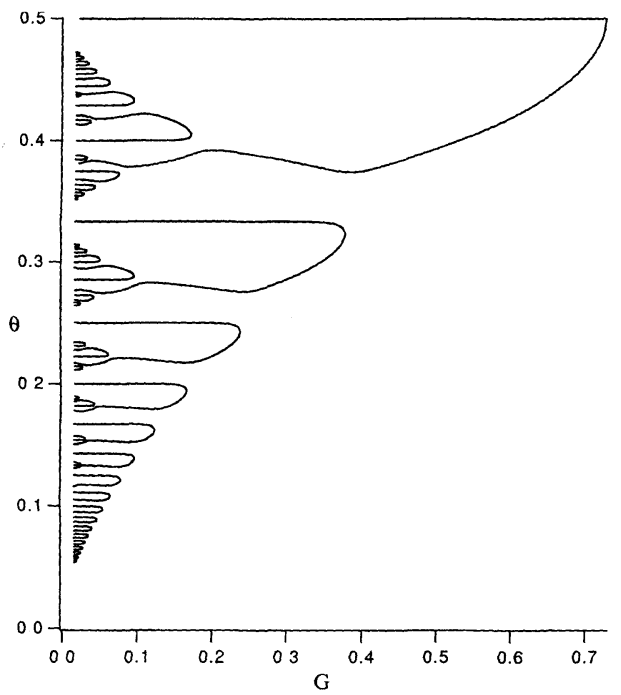

Fig. 13. The spectrum $\Delta$ with the condition of Levitov. The family of spiral lattices is $\operatorname{Se}(G, \theta)$ and $-\frac{1}{r} \frac{d u}{d r}=e^{-K r}, K=10$

We will restrict ourselves to the case of linear spiral lattices but the results can be generalized to other cases. Sometimes constant factors will be omitted in the formula and some calculations will not be made, in order to lighten the exposition.

Let us recall that $H_{K}(G, \theta)=\sum_{n \geqq 1} f_{K}\left(\left\|\mathbf{R}_{\sigma(n)}(G, \theta)\right\|\right) \mathbf{R}_{\sigma(n)} \cdot \mathbf{e}_{y}$ and $\Delta_{K}=$ $\left\{(G, \theta): H_{K}(G, \theta)=0\right\}$. If $f_{K}(r)=r^{-K}$, one has

Lemma 13. Let $0<\mu<0.1$ and define

$$
\Omega_{ \pm}\left(\frac{p}{q}\right)=\left\{\left(G, \frac{p}{q} \pm G\right): 0<G<\frac{\mu}{q^{2}}\right\} .
$$

There exists $K_{0}$ such that $\forall K>K_{0}, \forall \frac{p}{q} \in \mathbb{Q}$

$$
\pm H_{K}(G, \theta)>0, \forall(G, \theta) \in \Omega_{ \pm}\left(\frac{p}{q}\right) .
$$

Lemma 14. Consider the set

$$
\Sigma^{*}(\alpha, \beta)=\Sigma(\alpha) \cup \bigcup_{\frac{p}{q} \in \mathbb{Q} \cap[0,1]}^{\bigcup} X_{p / q}(\beta),
$$

where

$$
\begin{gathered}
\Sigma(\alpha)=\bigcup_{(G, \theta) \in \Sigma} B((G, \theta) ; \alpha G) \\
X_{p / q}(\beta)=\bigcup_{(G, \theta) \in\left(\Sigma^{*} \backslash \Sigma\right) \cap \Sigma_{p / q}}\left((G, \theta) ; \frac{\beta \sqrt{G}}{q}\right)
\end{gathered}
$$

and $B((G, \theta) ; t)$ is the open ball of center $(G, \theta)$ and radius $t$. 


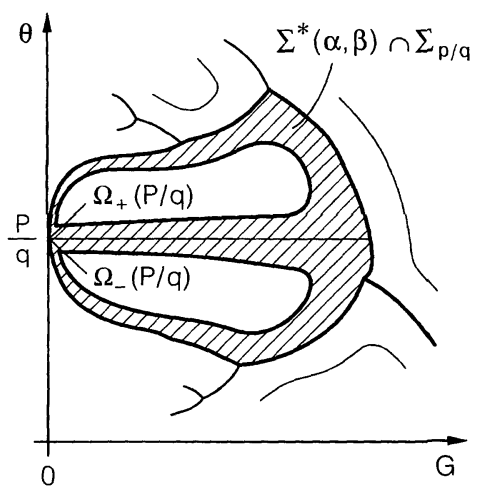

Fig. 14. $\Sigma^{*}(\alpha, \beta)$ is obtained by constructing an open ball on every point of $\Sigma^{*} . \alpha$ and $\beta$ can be chosen so that $\Omega_{+}\left(\frac{p}{q}\right)$ and $\Omega_{-}\left(\frac{p}{q}\right)$ cross the boundary of $\left.\Sigma^{*}(\alpha, \beta), \forall p / q \in \mathbb{Q} \cap\right] 0,1[$

If $\alpha, \beta>0$ are small enough, then

1) $\forall p / q \in \mathbb{Q} \cap] 0,1[$, the boundary of the set

$$
\Sigma^{*}(\alpha, \beta) \cap \Sigma_{p / q}
$$

is the union of the boundary of $\Sigma_{p / q}$ and two simple closed curves. These two curves intersect the segments $\Omega_{+}\left(\frac{p}{q}\right)$ and $\Omega_{-}\left(\frac{p}{q}\right)$ of Lemma 13 .

The situation is illustrated in Fig. 14.

$$
\begin{gathered}
\sup _{(G, \theta) \notin \Sigma^{*}(\alpha, \beta)} \frac{\left\|\mathbf{R}_{\sigma(1)}\right\|}{\left\|\mathbf{R}_{\sigma(2)}\right\|}=B_{1}<1 . \\
\sup _{(G, \theta) \notin \Sigma^{*}(\alpha, \beta)} \frac{\left\|\mathbf{R}_{\sigma(1)}\right\|}{\left|\mathbf{R}_{\sigma(1)} \cdot \mathbf{e}_{y}\right|}=B_{2}<\infty .
\end{gathered}
$$

The proofs of Lemma 13 and 14 are given in Appendix B. The following theorem is the analoguous of Theorem 11:

Theorem 15. $\forall \alpha, \beta>0$ there exists $K_{0}>0$ such that if $K>K_{0}$,

$$
\Delta_{K} \subset \Sigma^{*}(\alpha, \beta) \text {. }
$$

Proof. Suppose that $(G, \theta) \notin \Sigma^{*}(\alpha, \beta)$. Let us prove that if $K$ is great enough,

$$
1>\sum_{n \geqq 2}\left|\frac{f_{k}\left(R_{\sigma(n)}\right) \mathbf{R}_{\sigma(n)} \cdot \mathbf{e}_{y}}{f_{k}\left(R_{\sigma(1)}\right) \mathbf{R}_{\sigma(1)} \cdot \mathbf{e}_{y}}\right|=\sum_{n \geqq 2}\left|\frac{\mathbf{R}_{\sigma(n)} \cdot \mathbf{e}_{y}}{\mathbf{R}_{\sigma(1)} \cdot \mathbf{e}_{y}}\right|\left(\frac{R_{\sigma(1)}}{R_{\sigma(n)}}\right)^{K} .
$$

But

$$
\begin{aligned}
& \sum_{n \geqq 2}\left|\frac{\mathbf{R}_{\sigma(n)} \cdot \mathbf{e}_{y}}{\mathbf{R}_{\sigma(1)} \cdot \mathbf{e}_{y}}\right|\left(\frac{R_{\sigma(1)}}{R_{\sigma(n)}}\right)^{K} \leqq \frac{R_{\sigma(1)}}{\left|\mathbf{R}_{\sigma(1)} \cdot \mathbf{e}_{y}\right|} \sum_{n \geqq 2} \frac{R_{\sigma(n)}}{R_{\sigma(1)}}\left(\frac{R_{\sigma(1)}}{R_{\sigma(n)}}\right)^{K} \\
& =\frac{R_{\sigma(1)}}{\left|\mathbf{R}_{\sigma(1)} \cdot \mathbf{e}_{y}\right|} \cdot O\left(\left(\frac{R_{\sigma(1)}}{R_{\sigma(2)}}\right)^{K-3}\right) .
\end{aligned}
$$

The conclusion follows from Lemma 14. 


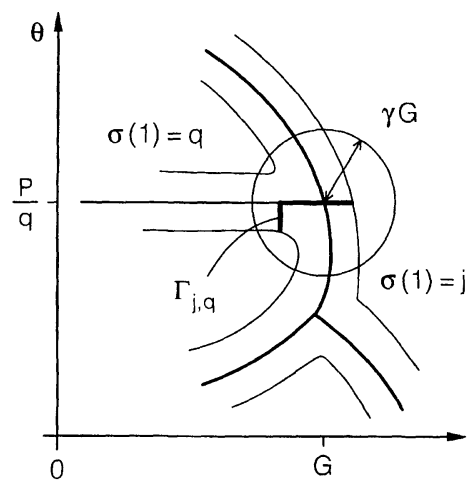

Fig. 15. $\Gamma_{j, q}$ is included in an open ball of radius $\gamma G$ and center $(G, p / q) \in \Sigma$

In the next theorem one constructs a set of lines $\Gamma_{j, q}$; each line $\Gamma_{j, q}$ crosses a singular branch $(j, q)$ in $\Sigma$, divides $\Sigma^{*}(\alpha, \beta)$ in two parts and contains no point of $\Delta_{K}$. The main difference with Theorem 12 lies in the fact that this construction is done for an infinity of singular branches.

Theorem 16. Let $C \in] 1,2\left[\right.$. Let $K_{0}$ be such that $\Delta_{K} \subset \Sigma^{*}(\alpha, \beta)$ if $K>K_{0}$. If $(j, q)$ is a singular branch of $\Sigma$, one constructs the broken line $\Gamma_{j, q}$ in exactly the same way as $\Gamma$ (cf. Theorem 12 and Fig. 8 ): $\Gamma_{j, q}$ is included in $\Sigma^{*}(\alpha, \beta)$, crosses $(j, q)$ and divides $\Sigma^{*}(\alpha, \beta)$ in two parts.

If $\alpha, \beta$ are small enough and $K_{0}$ is large enough, then

$$
\Delta_{K} \cap \Gamma_{j, q}=\varnothing
$$

for any $K>K_{0}$ and any $(j, q)$ such that $\frac{2(q-j)}{q}>C$.

Proof. Let $(j, q)$ be a singular branch such that $\frac{2(q-j)}{q}>C$. There exists a constant $\gamma$, independent of $j$ and $q$, such that $\Gamma_{j, q}$ is included in a ball of radius $\gamma G$ and center $(G, p / q) \in \Sigma$ (see Fig. 15). To see this observe that $\frac{\beta \sqrt{G}}{q} \approx \beta G$ near of $(G, p / q)$; moreover in the neighbourhood of $(G, p / q)$ the slope $\frac{d G}{d \theta}$ of the singular branch $(j, q)$ is uniformly bounded (direct calculation). Now if $\alpha$ and $\beta$ are small enough, $\gamma$ can be chosen small enough for Proposition 20 of Appendix B to be applied: $\frac{R_{\sigma(3)}}{R_{\sigma(2)}}>\sqrt{C}-\gamma>1$ on $\Gamma_{j, q}$.

Let us prove that $H_{K}$ can not cancel on $\Gamma_{j, q}$ if $K$ is great enough. On one hand

$$
\left|\sum_{n \geqq 3}\left(\frac{R_{\sigma(2)}}{R_{\sigma(i)}}\right)^{K} q \mathbf{R}_{\sigma(i)} \cdot \mathbf{e}_{y}\right|=O\left(\left(\frac{R_{\sigma(2)}}{R_{\sigma(3)}}\right)^{K-2}\right),
$$

and on the other hand

$$
\left|\left(\frac{R_{\sigma(2)}}{R_{\sigma(1)}}\right)^{K} \mathbf{R}_{\sigma(1)} \cdot \mathbf{e}_{y}+\mathbf{R}_{\sigma(2)} \cdot \mathbf{e}_{y}\right|>\sin \frac{2 \pi}{q}
$$

(cf. Theorem 12). The conclusion follows. 
Let us examine the consequences of Theorems 15 and 16. Consider a singular branch $(j, q)$ containing a point $\left(G, p / q=\left[a_{1}, \ldots, a_{n}\right]\right)\left(a_{n}>1\right)$. The condition $\frac{2(q-j)}{q}>C$ is equivalent to

$$
a_{n}-1+\frac{1}{a_{n-1}+\frac{1}{a_{n-2}+\ldots}}>\frac{C}{2-C} .
$$

Consider now the path in $\Sigma^{*}$ that goes from $\left(G=0, \theta=p / q=\left[a_{1}, \ldots, a_{n}\right]\right)$ to the singular branch $(j, q)$ and then along successive regular branches of $\Sigma$. This is the unique path in $\Sigma^{*}$ crossing $\Sigma_{p / q}$ and connecting $\left(G=0, \theta=\left[a_{1}, \ldots, a_{n}\right]\right)$ with $\left(G=0, \theta=\left[a_{1}, \ldots, a_{n}, 1,1,1, \ldots\right]\right)$. Let $C, \alpha, \beta, K_{0}$ be such that Theorem 16 holds. It is possible to make the construction of Theorem 16 on each singular branch connected to this path if

$$
\frac{2}{2-C}-\frac{1}{a_{n-1}+\frac{1}{a_{n-2}+\ldots}}<a_{n}<\frac{2-C}{2 C-2}-\frac{1}{a_{n-1}+\frac{1}{a_{n-2}+\ldots}}
$$

(this can be directly verified using (3.23) and the properties of $\Sigma$ ). Equation (3.24) has no solution if $C>1.17$.. But if $C>1$ is taken small enough, the condition (3.24) reduces to an upper bound on $a_{n}$ and an upper bound on $a_{n-1}$ if $a_{n}=2$. These bounds tend towards $+\infty$ as $C$ tends towards 1 .

If the condition (3.24) is fulfilled one can therefore construct a tongue along the half-loop in $\Sigma^{*}$ connecting $\left(G=0, \theta=\left[a_{1}, \ldots, a_{n}\right]\right)$ with $\left(G=0, \theta=\left[a_{1}, \ldots, a_{n}\right.\right.$, $1,1,1, \ldots])$; the continuous lines of $\Delta_{K}$ are contained in such tongues and in the rest of $\Sigma^{*}(\alpha, \beta)$. This enables us to prove the following:

Theorem 17. Let $C \in] 1,1.17 . .\left[\right.$. Let $\alpha, \beta, K_{0}$ be such that the conditions of Theorems 15 and 16 are satisfied. If $p / q=\left[a_{1}, \ldots, a_{n}\right]\left(a_{n}>1\right)$ satisfies (3.24), then $\forall K>K_{0}$ there exists a continuous line belonging to $\Delta_{K}$ and connecting $\left(G=0, \theta=\left[a_{1}, \ldots, a_{n}\right]\right)$ with $\left(G=0, \theta=\left[a_{1}, \ldots, a_{n}, 1,1,1, \ldots\right]\right)$.

Proof. Consider as above the unique path in $\Sigma^{*}$ crossing $\Sigma_{p / q}$ and connecting $(G=$ $\left.0, \theta=\left[a_{1}, \ldots, a_{n}\right]\right)$ with $\left(G=0, \theta=\left[a_{1}, \ldots, a_{n}, 1,1,1, \ldots\right]\right)$. By virtue of Lemmas 13 and 14 and Theorems 15 and 16, there exists two continuous lines on each side of this path, connecting the same points on the axis $G=0$, on which $H_{K}(G, \theta)$ does not cancel (see Fig. 16). By Lemma 13 the sign of $H_{K}(G, \theta)$ is different on each line. This proves the theorem.

3.3. The Absolute Minimality Condition in the Geometric Limit. We have described so far a spectrum defined by an extremality condition instead of the original minimality condition, which greatly simplified the matter. The problem becomes especially difficult if one considers the absolute minimality condition of Douady and Couder (see $[19,20]$ ). However a simple result can be obtained for the simple periodic lattice $W(G, \theta)$ in the limit of infinitely short-range interaction.

Consider for instance the interaction

$$
u_{K}(r)=e^{-K r}, K \geqq 0
$$




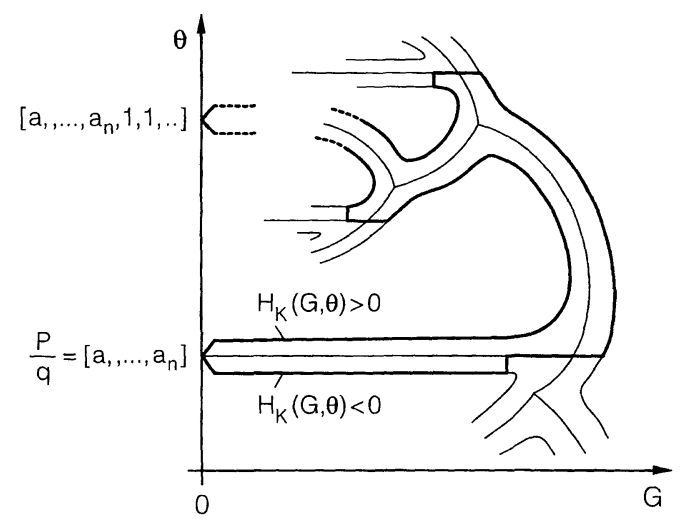

Fig. 16. By virtue of Lemmas 13 and 14 and Theorems 15 and 16 , one can construct two continuous lines connecting $\left(G=0, \theta=\left[a_{1}, \ldots, a_{n}\right]\right)$ and $\left(G=0, \theta=\left[a_{1}, \ldots, a_{n}, 1,1,1, \ldots\right]\right)$ such that $H_{K}(G, \theta)$ has a different sign on each line

and construct the resulting potential (cf. (1.6))

$$
U_{K}(x)=\sum_{n \in \mathbb{N}^{*}, m \in \mathbb{Z}} u_{K}\left(\left\|x \mathbf{e}_{x}-\mathbf{r}_{n m}\right\|\right)
$$

In the limit $K \rightarrow+\infty$ the minimality condition

$$
U_{K}(x)-U_{K}(0) \geqq 0, \forall x
$$

reduces to

$$
d_{G, \theta}(0) \geqq d_{G, \theta}(x), \forall x,
$$

where

$$
d_{G, \theta}(x)=\min _{n \in \mathbb{N}^{*}, m \in \mathbb{Z}}\left\|x \mathbf{e}_{x}-\mathbf{r}_{n m}(G, \theta)\right\| .
$$

It is not difficult to convince oneself that the spectrum defined by (3.28) is a subset of $\Sigma$. Moreover there is always a part of the singular branch $(n, n+m)$, connected to $(n, m)$, that is not included in this spectrum. The following theorem completes its description.

Theorem 18. If $(G, \theta)$ belongs to any regular branch of $\Sigma$, then

$$
d_{G, \theta}(0) \geqq d_{G, \theta}(x), \forall x .
$$

Thus in the limit $K \rightarrow+\infty$, the spectrum defined by the extremality condition of Douady and Couder is basically the set of the regular branches of $\Sigma$. It clearly shows the expected selection of noble numbers.

Proof of Theorem 18. Suppose that $(G, \theta)$ belongs to a regular branch of $\Sigma$. This implies

$$
\left(\mathbf{R}_{\sigma(1)} \cdot \mathbf{e}_{x}\right)\left(\mathbf{R}_{\sigma(2)} \cdot \mathbf{e}_{x}\right)<0
$$


Assume that $\sigma(2)>\sigma(1)$. We will prove that if

$$
d_{G, \theta}(x)>\left\|\mathbf{R}_{\sigma(1)}\right\|=d_{G, \theta}(0)
$$

for some $x$, then $\sigma(2)>2 \sigma(1)$, which is a contradiction.

There exists $\mathbf{a} \in W(G, \theta)$ such that the unit cell

$$
\left\{\mathbf{a}, \mathbf{a}+\mathbf{R}_{\sigma(1)}, \mathbf{a}+\mathbf{R}_{\sigma(2)}, \mathbf{a}+\mathbf{R}_{\sigma(1)}+\mathbf{R}_{\sigma(2)}\right\}
$$

contains the point $x \mathbf{e}_{x}$. One can distinguish three cases:

1) $\left(\mathbf{a}+\mathbf{R}_{\sigma(1)}\right) \cdot \mathbf{e}_{y}>0$ and $\mathbf{a} \cdot \mathbf{e}_{y} \leqq 0$.

In this case it is easy to see that $d_{G, \theta}(x) \leqq\left\|\mathbf{R}_{\sigma(1)}\right\|$.

2) $\left(\mathbf{a}+\mathbf{R}_{\sigma(2)}\right) \cdot \mathbf{e}_{y} \leqq 0$.

Idem.

3) $\left(\mathbf{a}+\mathbf{R}_{\sigma(1)}\right) \cdot \mathbf{e}_{y} \leqq 0$ and $\left(\mathbf{a}+\mathbf{R}_{\sigma(2)}\right) \cdot \mathbf{e}_{y}>0$.

$d_{G, \theta}(x)$ takes its greatest value if $\left(\mathbf{a}+\mathbf{R}_{\sigma(1)}\right) \cdot \mathbf{e}_{y}=0$. Without loss of generality, one can therefore assume that $\mathbf{a}+\mathbf{R}_{\sigma(1)}=0$. One seeks $x$ such that $d_{G, \theta}(x)>$ $\left\|\mathbf{R}_{\sigma(1)}\right\|$. The function $d_{G, \theta}(x)$ has a maximum that can be greater than $\left\|\mathbf{R}_{\sigma(1)}\right\|$ at $x=\tilde{x}$, where:

$$
\left\|\tilde{x} \mathbf{e}_{x}-\left(\mathbf{a}+\mathbf{R}_{\sigma(2)}\right)\right\|=\left\|\tilde{x} \mathbf{e}_{x}-\left(\mathbf{a}+\mathbf{R}_{\sigma(1)}+\mathbf{R}_{\sigma(2)}\right)\right\|
$$

Suppose that

$$
\left\|\mathbf{R}_{\sigma(1)}\right\|<d_{G, \theta}(\tilde{x}) \leqq\left\|\tilde{\mathbf{x}} \mathbf{e}_{x}-\left(\mathbf{a}+\mathbf{R}_{\sigma(2)}\right)\right\| .
$$

Combining (3.33) and (3.34) with the hypothesis $\left\|\mathbf{R}_{\sigma(1)}\right\|=\left\|\mathbf{R}_{\sigma(2)}\right\|$, one obtains

$$
\mathbf{R}_{\sigma(1)}{ }^{2} \mathbf{R}_{\sigma(2)}{ }^{2}>4\left(\left(\mathbf{R}_{\sigma(1)} \cdot \mathbf{e}_{y}\right)\left(\mathbf{R}_{\sigma(2)} \cdot \mathbf{e}_{y}\right)-\left(\mathbf{R}_{\sigma(1)} \cdot \mathbf{e}_{x}\right)\left(\mathbf{R}_{\sigma(2)} \cdot \mathbf{e}_{x}\right)\right) \text {. }
$$

In other words, if $\alpha$ is the angle between $\mathbf{R}_{\sigma(1)}$ and $\left(-\mathbf{R}_{\sigma(2)} \cdot \mathbf{e}_{x}, \mathbf{R}_{\sigma(2)} \cdot \mathbf{e}_{y}\right)$, one has

$$
\alpha>\pi / 3 \text {. }
$$

Consequently

$$
\frac{\mathbf{R}_{\sigma(2)} \cdot \mathbf{e}_{y}}{\mathbf{R}_{\sigma(1)} \cdot \mathbf{e}_{y}}=\frac{\sigma(2)}{\sigma(1)}>\min _{0 \leqq \beta \leqq \pi / 6} \frac{\sin (\beta+\pi / 3)}{\sin \beta}=2,
$$

which had to be proved.

Adler [18] and Levitov [8] obtained an analogous result with the relative minimality condition for the variational problem (in the paper of Adler the role of energy is played by contact pressure).

\section{Conclusion}

Following [8-10], we have studied systems of particles interacting repulsively through a potential $u(r)$ and arranged in a periodic or a spiral plane lattice. We considered families of lattices depending on two parameters, $G$ and $\theta, G$ is related to the vertical or radial compression of the lattice $\theta$ is called the divergence 
of the lattice. The divergence spectrum $\Delta$ was defined as the set of couples $(G, \theta)$ which satisfy the extremality condition (1.5) or (1.17). The first one corresponds to the dynamical condition of Douady and Couder (one seeks the lattices that are the stationary solutions of a discrete time dynamical system) while the second one is the variational problem of Levitov. Formally both conditions are essentially equivalent.

Levitov solved the variational problem for the periodic lattice. He showed that this particular problem possesses a hidden symmetry, so that the spectrum is usually constituted of a set of branches arranged in the Farey structure; each branch cuts the axis $G=0$ at a noble number. Using the dynamical condition, Douady and Couder obtained numerically a similar spectrum for spiral lattices.

We addressed the following question: apart from the case treated by Levitov, where the noble numbers appear a consequence of some symmetry, under what assumptions can one prove that the spectrum $\Delta(G, \theta)$ shows the hierarchical selection of noble numbers put forward by Levitov and Douady and Couder?

As these authors indicated, the selection of noble numbers is due to two main facts:

- there is an underlying geometrical problem, which is in a way the unperturbed problem. Its solution possesses the Farey tree structure. This establishes a direct connection between the spectrum $\Delta$ and the continued fraction representation of the divergence $\theta$.

- the physical extremality condition results in the choice of the so-called "regular branch" at each bifurcation in the Farey tree. Thus ideally every possible path in the perturbed tree leads to a noble number. The tree structure organizes noble numbers into a natural hierarchy.

In Sect. 2.3 we dealt with the geometrical problem in the case of a class of spiral lattices (the case of the periodic lattice being well known). We proved that the solution has basically the Farey tree structure: there is at the most a finite number of defects in the tree. In the special case of a linear or an exponential spiral lattice, a numerical calculation shows that there are no such defects.

In Sect. 3.1 we considered the divergence spectrum $\Delta$ in the case of an exponential interaction $u(r) \sim e^{-K r}$. We proved that the branches of the spectrum are included in tongues of width $\varepsilon(K)$. These tongues follow the paths in the Farey tree that lead to noble numbers (more precisely, they connect a rational number with the corresponding noble number on the axis $G=0) . \varepsilon(K)$ tends towards zero as $K$ tends towards infinity, so that noble numbers are approached with an increasing accuracy if the range of the interaction is decreased. On the other hand, this structure of the spectrum is destroyed when $G$ is smaller than some characteristic value, that is when the shortest distance between two lattice points is much smaller than the characteristic length of the potential (the nearest neighbours approximation (1.13) loses all its relevance in this case). One can thus construct counterexamples in which this structure of the spectrum entirely disappears.

If the interaction is scale invariant $\left(u(r)=r^{-K}\right)$, no characteristic value of $G$ can be defined. In this case, we proved in Sect. 3.2 that there exists a $K_{0}$ such that if $K>K_{0}$, a class of noble numbers is exactly selected. A similar result must be obtained for other potentials if the density of the lattice is kept constant (or is increased) when $G$ is decreased.

These results contrast with the situation analysed by Levitov, where the structure of the spectrum is essentially independent of the potential. The following table shows the accuracy of the selection of noble number according to the different cases: 


$$
u(r) \sim e^{-K r} \quad u(r) \sim r^{-K}
$$

Douady-Couder condition :

Spiral lattice

\begin{tabular}{l} 
Levitov \\
condition : \\
\hline $\begin{array}{l}\text { Douady-Couder } \\
\text { condition: }\end{array}$
\end{tabular}

Periodic lattice

$$
\begin{array}{ll}
\text { finite accuracy } & \text { infinite accuracy } \\
\text { depending on } K & \text { if } K>K_{0}
\end{array}
$$

finite accuracy infinite accuracy depending on $K$ if $K>K_{0}$

$$
u(r) \sim e^{-K r} \quad u(r) \sim^{-K}
$$

\section{Levitov \\ condition : infinite accuracy infinite accuracy $\forall K \quad \forall K$}

It is well worth noting that spectrum originally considered in $[8,9]$ and $[10]$ was defined by a minimality condition (instead of an extremality condition). It is therefore a subset of the spectrum we studied, from which the plateaus around the rational divergences usually disappear; the branches may also become discontinuous $[19,20]$. In Sect. 3.3, we examined the spectrum defined by the absolute minimality condition of Douady and Couder in the geometric limit (for instance $\left.u(r)=e^{-K r}, K \rightarrow+\infty\right)$. In the case of the periodic lattice, it is constituted by the same hierarchical set of branches leading to noble numbers.

Ultimately, then, the hierarchical selection of noble numbers in these problems is closely linked to the two-dimensional geometry of the system. This fact is interesting as regards the problem of phyllotaxis. The systems we studied were proposed as possible examples of the mechanism controlling the appearance of new leaves on the stem. Equivalent mechanisms can indeed be associated with one-dimensional systems (for instance reaction and diffusion of chemical substances on the meristem $[13,16,17,25])$. In this more general framework, Guerreiro $[19,20]$ showed that the mechanism itself fails to explain the universality of noble numbers in nature, unless it is considered in an appropriate limit.

Acknowledgements. The author wishes to express his thanks to F. Rothen for his critical reading of the manuscript. He is also indebted to J. Guerreiro and G.P. Bernasconi for many valuable suggestions.

\section{APPENDIX A.}

Proposition 6. (Sect. 2.3). Suppose that $\frac{d^{2} \Phi}{d x^{2}}(x) \geqq 0, \forall x \geqq 0$. If $t \leqq 1, T_{\Phi}^{-1}\left(\Omega_{t}\right)$ is a convex set.

Proof. The boundary of $\Omega_{t}$ is the union of two arcs of a circle. One is an arc of the unit circle and it is mapped by $T_{\Phi}^{-1}$ on a segment lying on the $x$-axis. The other arc is described in polar coordinates $(r, \varphi)$ by:

$$
r(\varphi)=\cos \varphi+\sqrt{t-\sin ^{2} \varphi},-\varphi_{\max } \leqq \varphi \leqq \varphi_{\max },
$$


where

$$
\varphi_{\max }=\arccos \left(1-t^{2} / 2\right) .
$$

$T_{\varphi}^{-1}$ maps this arc on a curve described in cartesian coordinates $(x, y)$ by :

$$
x=\varphi, y=h(\varphi),-\varphi_{\max } \leqq \varphi \leqq \varphi_{\max },
$$

where

$$
h(\varphi)=\Phi^{-1}(r(\varphi))
$$

We have to prove that

$$
h^{\prime \prime}(\varphi)=\frac{1}{\left(\Phi^{\prime} \circ h(\varphi)\right)^{2}}\left\{r^{\prime \prime}(\varphi) \Phi^{\prime} \circ h(\varphi)-r^{\prime 2}(\varphi) \frac{\Phi^{\prime \prime} \circ h(\varphi)}{\Phi^{\prime} \circ h(\varphi)}\right\} \leqq 0 .
$$

It is enough to verify that $r^{\prime \prime}(\varphi)<0$, which is easily seen if $t \leqq 1$.

Proposition 7. (Sect. 2.3). Suppose that

$$
\frac{d \log \Phi_{G}(n)}{d G}<\frac{d \log \Phi_{G}(m)}{d G}, \forall 1 \leqq n<m, \forall G \geqq 0 .
$$

Consider the function

$$
A_{n m}(G, \theta)=\mathbf{R}_{n}^{2}(G, \theta)-\mathbf{R}_{m}^{2}(G, \theta), n<m .
$$

For any fixed value of $\theta$ it has at the most one zero $G_{0}$; it is negative for $G>G_{0}$ and positive otherwise.

Proof. Let us show that if $A_{n m}(G, \theta)=0$, then $\partial_{G} A_{n m}(G, \theta) \neq 0$. First notice that

$$
A_{n m}=\Phi_{G}^{2}(n)-\Phi_{G}^{2}(m)-2\left(\Phi_{G}(n) \cos (2 \pi n \theta)-\Phi_{G}(m) \cos (2 \pi m \theta)\right) .
$$

Thus if $n<m$ and $A_{n m}(G, \theta)=0$, one has

$$
\Phi_{G}(m) \cos (2 \pi m \theta)-\Phi_{G}(n) \cos (2 \pi n \theta)>0 .
$$

Now if $A_{n m}(G, \theta)=0$ and $\partial_{G} A_{n m}(G, \theta)=0$, the following equality would hold:

$$
\frac{\frac{d \Phi_{G}(n)}{d G}}{\frac{d \Phi_{G}(m)}{d G}}=\frac{\Phi_{G}(n)}{\Phi_{G}(m)}+\frac{\Phi_{G}(m) \cos (2 \pi m \theta)-\Phi_{G}(n) \cos (2 \pi n \theta)}{\Phi_{G}(m)\left(\Phi_{G}(n)-\cos (2 \pi n \theta)\right)} .
$$

It would clearly contradict the assumption

$$
\frac{d \log \Phi_{G}(n)}{d G}<\frac{d \log \Phi_{G}(m)}{d G}, \quad \forall n<m, \forall G \geqq 0 .
$$

Consequently, the sign of $\partial_{G} A_{n m}(G, \theta)$ does not change along an implicit curve defined by $A_{n m}(G, \theta)=0$ in the $(G, \theta)$ plane. In fact if $n<m, \partial_{G} A_{n m}(G, \theta)<0$ along such a curve, which demonstrates the proposition. To prove this last inequality, notice that such a curve necessarily cuts the axis $G=0$; now at this point

$$
\left.\partial_{G} A_{n m}(0, \theta)=2(1-\cos (2 \pi n \theta)) \frac{d\left(\Phi_{G}(n)-\Phi_{G}(m)\right)}{d G}\right)_{G=0}<0
$$

by hypothesis. 


\section{APPENDIX B.}

Lemma 13. (Sect. 3.2). Let $0<\mu<0.1$ and define

$$
\Omega_{ \pm}\left(\frac{p}{q}\right)=\left\{\left(G, \frac{p}{q} \pm G\right): 0<G<\frac{\mu}{q^{2}}\right\} .
$$

There exists $K_{0}$ such that $\forall K>K_{0}, \forall \frac{p}{q} \in \mathbb{Q}$

$$
\pm H_{K}(G, \theta)>0, \forall(G, \theta) \in \Omega_{ \pm}\left(\frac{p}{q}\right)
$$

Proof. Let us write

$$
H_{K}(G, \theta)=\sum_{n \geqq 1} f_{K}\left(\left\|\mathbf{R}_{n q}\right\|\right) \mathbf{R}_{n q} \cdot \mathbf{e}_{y}+\sum_{n \geqq 0} \sum_{l=1}^{q-1} f_{K}\left(\left\|\mathbf{R}_{n q+1}\right\|\right) \mathbf{R}_{n q+1} \cdot \mathbf{e}_{y} .
$$

If $(G, \theta) \in \Omega_{ \pm}\left(\frac{p}{q}\right)$, it is not difficult to convince oneself that the second sum becomes negligible if $K$ is great enough. As a matter of fact, the leading term of the second sum is approximately equal to $q^{K-1}$, whereas the leading term of the first sum is approximately $(q G)^{1-K}>(10 q)^{K-1}$.

Lemma 14. (Sect. 3.2). Consider the set

$$
\Sigma^{*}(\alpha, \beta)=\Sigma(\alpha) \cup \bigcup_{\frac{p}{q} \in \mathbb{Q} \cap[0,1]} X_{p / q}(\beta),
$$

where

$$
\begin{gathered}
\Sigma(\alpha)=\bigcup_{(G, \theta) \in \Sigma} B((G, \theta) ; \alpha G) \\
X_{p / q}(\beta)=\bigcup_{(G, \theta) \in\left(\Sigma^{*} \backslash \Sigma\right) \cap \Sigma_{p / q}} B\left((G, \theta) ; \frac{\beta \sqrt{G}}{q}\right)
\end{gathered}
$$

and $B((G, \theta) ; t)$ is the open ball of center $(G, \theta)$ and radius $t$.

If $\alpha, \beta>0$ are small enough, then

1) $\forall p / q \in \mathbb{Q} \cap] 0,1[$, the boundary of the set

$$
\Sigma^{*}(\alpha, \beta) \cap \Sigma_{p / q}
$$

is the union of the boundary of $\Sigma_{p / q}$ and two simple closed curves. These two curves intersect the segments $\Omega_{+}\left(\frac{p}{q}\right)$ and $\Omega_{-}\left(\frac{p}{q}\right)$ of Lemma 13 .

The situation is illustrated in Fig. 14. of Sect. 3.2.

$$
\begin{gathered}
\sup _{(G, \theta) \notin \Sigma^{*}(\alpha, \beta)} \frac{\left\|\mathbf{R}_{\sigma(1)}\right\|}{\left\|\mathbf{R}_{\sigma(2)}\right\|}=B_{1}<1, \\
\sup _{(G, \theta) \notin \Sigma^{*}(\alpha, \beta)} \frac{\left\|\mathbf{R}_{\sigma(1)}\right\|}{\left|\mathbf{R}_{\sigma(1)} \cdot \mathbf{e}_{y}\right|}=B_{2}<\infty .
\end{gathered}
$$

Proof. Let us choose $G^{\prime}$ such that if $G<G^{\prime},\left\{\mathbf{r}_{\sigma(1)}, \mathbf{r}_{\sigma(2)}, \ldots, \mathbf{r}_{\sigma(10)}\right\} \subset \operatorname{Sl}(G, \theta)$ is a part of a simple periodic lattice as slightly distorted as we need. 
One can always construct the restriction of $\Sigma^{*}(\alpha, \beta)$ to the region $G^{\prime}<G<$ $G^{\prime \prime}<\infty$ (the complementary set is compact, so that it is easy to satisfy 2 ) and 3 )). Let us then restrict our attention to the region $G<G^{\prime}$, and assume that $\operatorname{Sl}(G, \theta)$ is a periodic lattice around $r_{0}$.

1) Consider a set $\Sigma_{p / q}$ as illustrated on Fig. 17. As G tends towards zero, $\sum_{p / q}$ is adjacent to sets of the type $\sum_{\frac{\tilde{j}+k p}{\tilde{j}+k q}}, k \rightarrow \infty$. One can deduce from this fact that $\delta(G) \approx \frac{\sqrt{G}}{q}$ as $G \rightarrow 0$ (if $\left(G, \frac{\tilde{p}+k p}{\tilde{q}+k p} \in \Sigma\right.$, then $G k q \approx \frac{1}{k q}$ and $\delta(G)=\frac{p}{q}-\frac{\tilde{p}+k p}{\tilde{q}+k p} \approx \frac{1}{k q^{2}}$ if $k$ is large).

It can be verified directly that the restriction of the boundary of $X_{p / q}(\beta)$ to the region $G<<\frac{1}{q^{2}}$ is the set of points

$$
\left(G, \frac{p}{q} \pm \frac{\beta}{q} \sqrt{G+\frac{\beta^{2}}{4 q^{2}}}\right) .
$$

As for the boundary of $\Sigma(\alpha) \cap \Sigma_{p / q}$, it is the union of the boundary $\Sigma_{p / q}$ and a simple closed curve. If $G<<\frac{1}{q^{2}}$ and $\alpha<<1$, this curve behaves basically like $\delta(G)$, so that it intersects the boundary of $X_{p / q}(\beta)$ at a point $(\tilde{G}, \tilde{\theta})$, where

$$
\tilde{G} \approx \frac{\beta^{2}}{4 q^{2}}, \tilde{\theta} \approx \frac{p}{q} \pm \frac{\beta^{2}}{q^{2}} .
$$

If $\beta^{2}<<\mu$ the boundary of $\Sigma^{*}(\alpha, \beta) \cap \Sigma_{p / q}$ must therefore intersect $\Omega_{+}\left(\frac{p}{q}\right)$ and $\Omega\left(\frac{p}{q}\right)$

2) If $(G, 0) \notin \Sigma(\alpha)$, then $\operatorname{dist}((G, 0), \Sigma)>\alpha G$. By Proposition 19 of this appendix, we know that

$$
\left(\frac{R_{\sigma(2)}^{2}}{R_{\sigma(1)}^{2}}-1\right) G>\alpha G .
$$

In other words, $B_{1} \cong \frac{1}{\sqrt{1+9}}$.

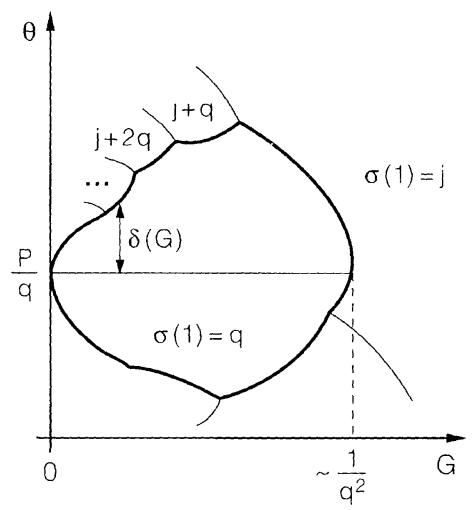

Fig. 17. The thick curve is the boundary of the set $\Sigma_{p / q}$ 
3) One can easily see that if $(G, \theta) \in \Sigma_{p / q} \backslash \Sigma^{*}(\alpha, \beta)$, G fixed,

$$
\left|\mathbf{R}_{\sigma(1)} \cdot \mathbf{e}_{y}\right| \geqq \beta \sqrt{G} \text {. }
$$

If $G$ is fixed, $\left\|\mathbf{R}_{\sigma(1)}\right\|(G, \theta)$ is maximum on the points of $\Sigma$. One can deduce from this that $\left\|\mathbf{R}_{\sigma(1)}\right\|=O(\sqrt{G})$. Hence

$$
\frac{\left\|\mathbf{R}_{\sigma(1)}\right\|}{\left|\mathbf{R}_{\sigma(1)} \cdot \mathbf{e}_{y}\right|}=\frac{O(\sqrt{G})}{\beta \sqrt{G}}=O(1) .
$$

Proposition 19. Let $W\left(G_{0}, \theta_{0}\right)$ be a simple periodic lattice and suppose that $\sigma(1)=n, \sigma(2)=m$ and $\frac{\left\|\mathbf{R}_{\sigma(2)}\right\|}{\left\|\mathbf{R}_{\sigma(1)}\right\|}<A<2$. There exists $\mathbf{x}=(\delta G, \delta \theta)$ such that

$$
\left\|\mathbf{R}_{n}\left(G_{0}+\delta G, \theta_{0}+\delta_{0}\right)\right\|=\left\|\mathbf{R}_{m}\left(G_{0}+\delta G, \theta_{0}+\delta \theta\right)\right\|
$$

and

$$
\|\mathbf{x}\|<C G \cdot\left(\frac{\mathbf{R}_{m}{ }^{2}\left(G_{0}, \theta_{0}\right)}{\mathbf{R}_{n}{ }^{2}\left(G_{0}, \theta_{0}\right)}-1\right),
$$

where $C$ is some constant ( $C$ depends on $A$ and $C \approx 1$ ).

Proof. Let us agree upon the following understanding:

$$
\left\|\mathbf{R}_{n}\right\|=\left\|\mathbf{R}_{n}\left(G_{0}, \theta_{0}\right)\right\|
$$

(idem for $m$ ). We look for a solution $\mathbf{x}$ of minimal norm to the problem:

$$
\left(\mathbf{R}_{n}+n \mathbf{x}\right)^{2}=\left(\mathbf{R}_{m}+m \mathbf{x}\right)^{2} .
$$

One obtains

$$
x=\frac{2\left(\mathbf{R}_{m}^{2}-\mathbf{R}_{n}^{2}\right)}{b+b^{\prime}}
$$

where

$$
\begin{aligned}
b & =\left\|m \mathbf{R}_{m}-n \mathbf{R}_{n}\right\|, \\
b^{\prime} & =\left\|m \mathbf{R}_{n}-n \mathbf{R}_{m}\right\| .
\end{aligned}
$$

Since $\left\|\mathbf{R}_{n}-\mathbf{R}_{m}\right\|>\left\|\mathbf{R}_{m}\right\|$ and $\left\|\mathbf{R}_{n}+\mathbf{R}_{m}\right\|>\left\|\mathbf{R}_{n}\right\|$, one can verify that

$$
\begin{aligned}
b+b^{\prime} & >\sup \left(|m-n| \cdot\left\|\mathbf{R}_{n}\right\|,|m-| m-n\|\cdot\| \mathbf{R}_{n}\|,|n-| m-n\| \cdot\left\|\mathbf{R}_{n}\right\|\right) \\
& >C(m+n)\left\|\mathbf{R}_{n}\right\|
\end{aligned}
$$

and

$$
\left\|\mathbf{R}_{n}\right\|<C^{\prime}(n+m) G,
$$

where $\mathrm{C}, C^{\prime}$ are some constants.

Proposition 20. Consider the lattice family $S l(G, \theta)$. Let $\left(G_{0}, p / q\right) \in \Sigma$, and $\sigma(1)=q, \sigma(2)=n$. If $2(q-n) / q>C$, where $1<C<2$, one can construct $a$ ball $\mathrm{B}$ of radius $\frac{\gamma}{3} G_{0}$ and center $\left(G_{0}, p / q\right)$, where $\gamma<\sqrt{C}-1$, such that:

$$
\frac{\left\|\mathbf{R}_{\sigma(3)}(G, \theta)\right\|}{\left\|\boldsymbol{R}_{\sigma(2)}(G, \theta)\right\|}>\sqrt{C}-\gamma>1
$$

for any $(G, \theta) \in B$. 
Proof. One can deduce from the equality

$$
\mathbf{R}_{q}^{2}=\mathbf{R}_{n}^{2}
$$

that

$$
\frac{\mathbf{R}_{\sigma(3)}^{2}}{\mathbf{R}_{\sigma(2)}^{2}}=\frac{\mathbf{R}_{q-n}^{2}}{\mathbf{R}_{q}^{2}}>\frac{2(q-n)}{q}>C
$$

Let $\mathbf{x}=(\delta G, \delta \theta)$. One has:

$$
\begin{aligned}
& \frac{\left\|\mathbf{R}_{\sigma(3)}\right\|}{\left\|\boldsymbol{R}_{\sigma(2)}\right\|}\left(G_{0}+\delta G, p / q+\delta \theta\right) \\
& \quad \geqq \sup \left(\frac{R_{q-n}-(q-n)\|\mathbf{x}\|}{R_{q}+q\|\mathbf{x}\|}, \frac{R_{q-n}-(q-n)\|\mathbf{x}\|}{R_{n}+n\|\mathbf{x}\|}\right)>\sqrt{C}-\gamma
\end{aligned}
$$

if $\|\mathbf{x}\|<\frac{\gamma}{3} G_{0}$. In fact

$$
\|\mathbf{x}\|<\frac{\gamma}{3} G_{0}<\frac{\gamma}{1+\sqrt{C}-\gamma} \cdot \frac{R_{q}}{q}<\frac{R_{q-n}-(\sqrt{C}-\gamma) R_{q}}{(q-n)+(\sqrt{C}-\gamma) q}
$$

(idem for the other term).

\section{References}

1. Hofmeister, W.: Allgemeine Morphologie der Gewachse, Handbuch der Physiologischen Botanik, 1 Engelman, Leipzig 405 (1868)

2. Snow, M., Snow, R.: Phil. Trans. Roy. Soc. London Ser. B 225, 63 (1935)

3. Schimper, K.F.: Geiger's Mag. für Pharm. 29, 1 (1830)

4. Braun, A.: Nova Acta Acad. Caesar Leop. Carol. 15, 197 (1831); Flora, Iena 18, 145 (1835)

5. Bravais, L. \& A.: Ann. Sci. Nat. second series 7, 42 (1837); ibid. 8, 11 (1837)

6. Rothen, F., Koch, A.J.: J. Phys. France 50, 633 (1989); ibid. 50, 1603 (1989)

7. Van Iterson, G.: Mathematische und Mikroskopisch-Anatomische Studien über Blattstellungen, Jena: Gustav Fischer, 1907

8. Levitov, L.S.: Phys. Rev. Lett. 66, 224 (1991)

9. Levitov, L.S.: Europhys. Lett. 14, 533 (1991)

10. Douady, S., Couder, Y.: Phys. Rev. Lett. 68, 2098 (1992); La Recherche 24, 26 (1993)

11. Schoute, J.C.: Recl. Trav. Bot. Néerl. 10, 153 (1913)

12. Richards, F.J.: Symp. Soc. Exp. Biol. 2, 217 (1948) Phil. Trans. R. Soc. B 235, 509 (1951)

13. Thornley, J.H.M.: Ann. Bot. 39, 491 (1975); ibid. 509

14. Mitchison, G.H.: Science 196, 270 (1977)

15. Veen, A.H., Lindenmayer, A.: Plant Physiol. 60, 127 (1977)

16. Marzec, C., Kappraff, J.: J. Theor. Biol. 103, 201 (1983)

17. Koch, A.J., Guerreiro, I., Bernasconi, G.P., Sadik, J.: J. Phys. I France 4, 187 (1994)

18. Adler, I.: J. Theor. Biol. 45, 1 (1974)

19. Guerreiro, J.: Thèse de Doctorat, Université de Lausanne (1993)

20. Guerreiro, J.: to be published in Physica D (1994)

21. Hardy, G.H., Wright, E.M.: An Introduction to the Theory of Numbers, 5th ed; Oxford: Cearendon Press, 1988

22. Khinchin, A.Ya.: Continued Fractions, Chicago, IL: The University of Chicago Press, 1961

23. Klein, F.: Elementary Mathematics from an Advanced Standpoint, New York Mc Millan, 1932

24. Coxeter, H.S.M.: Introduction to Geometry. New York: Wiley, 1969, p. 1600; J. Algebra 20, 167 (1972)

25. Bernasconi, G.P.: Physica D 70, 90 (1993) 
